\title{
HOST SELECTIVITY OF NEROCILA ORBIGNYI (GUERIN-MENEVILLE, 1832) (ISOPODA, CYMOTHOIDAE) WITH A RECORD OF A NEW HOST FROM THE SEA OF MARMARA (TURKEY)
} Ahmet ÖKTENER * and Jean Paul TRILLES **

* Sheep Research Station, Department of Fisheries, Çanakkele Street 7 km, Bandırma, Balıkesir, Turkey, TR-10200, ahmetoktener@yahoo.com, mothocya@hotmail.com, ORCID: 0000-0003-0858-0532.

** University of Montpellier, Montpellier, Auguste Broussonnet Street 163 France, F-34090, jp.trilles@cegetel.net

DOI: 10.2478/trser-2021-0003

KEYWORDS: Nerocila, Cymothoidae, host selectivity, Mugil cephalus, Sea of Marmara.

ABSTRACT

Nerocila orbignyi (Isopoda, Cymothoidae) is reported for the first time on Mugil cephalus Linnaeus, 1758 (Pisces, Mugilidae) from Bandırma Bay (the Sea of Marmara, Turkey) during 2020. This paper aims to present the morphological characters of male of $N$. orbignyi from Turkey. Hosts infested with $N$. orbignyi are commented according to taxonomical status (order, families), ecological behaviours (habitat selections, feeding habits, school-solitary), morphological characters (scale types) according to current records. It may be said that $N$. orbignyi has been reported more frequently on fish belonging to the Perciformes order and Mugilidae and Sparidae families. It may also be said that this parasite selects also the fishes with carnivorous, demersal, schools, and migratory character.

RÉSUMÉ: Sélectivité de l'hôte de Nerocila orbignyi (Guerin-Meneville, 1832) (Isopoda, Cymothoidae) avec un enregistrement d'un nouvel hôte de la mer de Marmara (Turquie).

Nerocila orbignyi (Isopoda, Cymothoidae) est signalée pour la première fois sur Mugil cephalus Linnaeus, 1758 (Poissons, Mugilidae) sur Bandırma Bay (la mer de Marmara, Turquie) au cours de 2020. L'article vise à présenter les caractères morphologiques du mâle de $N$. orbignyi de Turquie. Les hôtes infestés par $N$. orbignyi sont commentés selon leur statut taxonomique (ordre, familles), leurs comportements écologiques (choix de l'habitat, habitudes alimentaires, leurs caractères morphologiques (types d'échelle) selon les enregistrements actuels. On peut dire que $N$. orbignyi a été signalé plus fréquemment que les poissons appartenant à l'ordre des Perciformes et aux familles des Mugilidae et des Sparidae. On peut également dire que ce parasite sélectionne également les poisons en bancs en bancs et à caractère carnivore, démersal, et migrateour.

REZUMAT: Selectivitatea gazdei Nerocila orbignyi (Guerin-Meneville, 1832) (Isopoda, Cymothoidae) cu înregistrarea unei noi gazde din Marea Marmara (Turcia).

Nerocila orbignyi (Isopoda, Cymothoidae) este raportată pentru prima dată pe Mugil cephalus Linnaeus, 1758 (Pești, Mugilidae) din Golful Bandırma (Marea Marmara, Turcia) în 2020. Lucrarea își propune să prezinte caracterele morfologice ale masculului de $N$. orbignyi din Turcia. Gazdele infestate cu N. orbignyi sunt descrise în funcție de încadrarea taxonomică (ordin, familie), comportamentul ecologice (selecția habitatelor, obiceiurile de hrănire, independența față de banc), caracterele morfologice (tipurile de solzi) conform înregistrărilor actuale. Se poate spune că N. orbignyi a fost raportată mai frecvent pe peștii aparținând ordinului Perciformes și familiilor Mugilidae și Sparidae. Se poate spune, de asemenea, că acest parazit selectează și peștii cu caracter carnivor, demersal, social și migrator. 


\section{INTRODUCTION}

Fish parasites are important groups of biodiversity. Parasites are in balance with host fish in the natural environment. However, parasites can be harmful due to changes in environmental conditions such as intensive stocking, temperature, ph, etc., in fish farms. Fish parasites can even lead to mass mortalities on fish farms (Northcott et al., 1997; Öktener and Ünal, 2020). Sometimes, parasites can become active for various reasons and cause mass mortalities in natural environments. For example, Haplosporidium pinnae Catanese et al., 2018 caused the mass mortality in endangered Pinna nobilis (Linnaeus 1758) fan mussels from the Mediterranean Sea (Panarese et al., 2019). It is known that there are 257 fish species from the Sea of Marmara (Bilecenoğlu et al. 2014). However, there are not many studies on parasite diversity in fish.

Cymothoidae (Crustacea) are ectoparasitic isopods especially on the skin, bucal cavity, gill cavity, fins of hosts belonging to different marine, freshwater and brackish teleost fish (Trilles, 1994). Although Cymothoids are typically reported from teleost fish, there are some unusual associations such as sponges (Monod, 1933), jellyfish (Haswell, 1880), crustaceans (Lemos de Castro and Gomes Corrêa, 1982), chondrichthyans (Moreira and Sadowsky, 1978), amphibians (Stadler, 1972), snakes (Saravanakumar et al., 2012). While there are many reports of cymothoids from wild fishes, there are also reports from fish farms and aquaculture systems (Bragoni et al., 1983, 1984; Mladineo, 2002; Horton and Okamura, 2003; Čolak et al., 2018).

The cymothoid diversity of fishes should be more deeply investigated to better understand the biology of them. The present study aims to report a new host record for Nerocila orbignyi from Turkey. In addition, hosts of Nerocila orbignyi are examined according to family characteristics, habitat selections, feeding habits.

\section{MATERIAL AND METHODS}

109 individuals of Mugil cephalus Linnaeus, 1758 were collected by hand fishing rod from Bandırma Bay, the Sea of Marmara of Turkey during 2020. Parasites were fixed in $70 \%$ ethanol. Parasites were dissected using a Wild M5 stereo microscope. The dissected parts were mounted on slides in a glycerin-gelatine mounting medium. The appendages were drawn with the aid of a camera lucida (Olympus BH-DA). The photos were taken with the aid of Canon camera (EOS 1100D) attached to the microscope. Measurements were taken in millimeter $(\mathrm{mm})$ with a micrometric program (Pro-way). Scientific names, synonyms were checked with the WoRMS Editorial Board (2020). The information of feeding habits, habitat characteristics of the host were prepared according to Froese and Pauly (2019). Identifications and comparisons of parasites were performed according to Trilles (1975), and Bruce (1987).

\section{RESULTS AND DISCUSSION}

Isopoda Latreille, 1817

Cymothoidae Leach, 1818

Nerocila Leach, 1818

Nerocila orbignyi (Guérin-Méneville, 1832) (Figs. 1-3) 


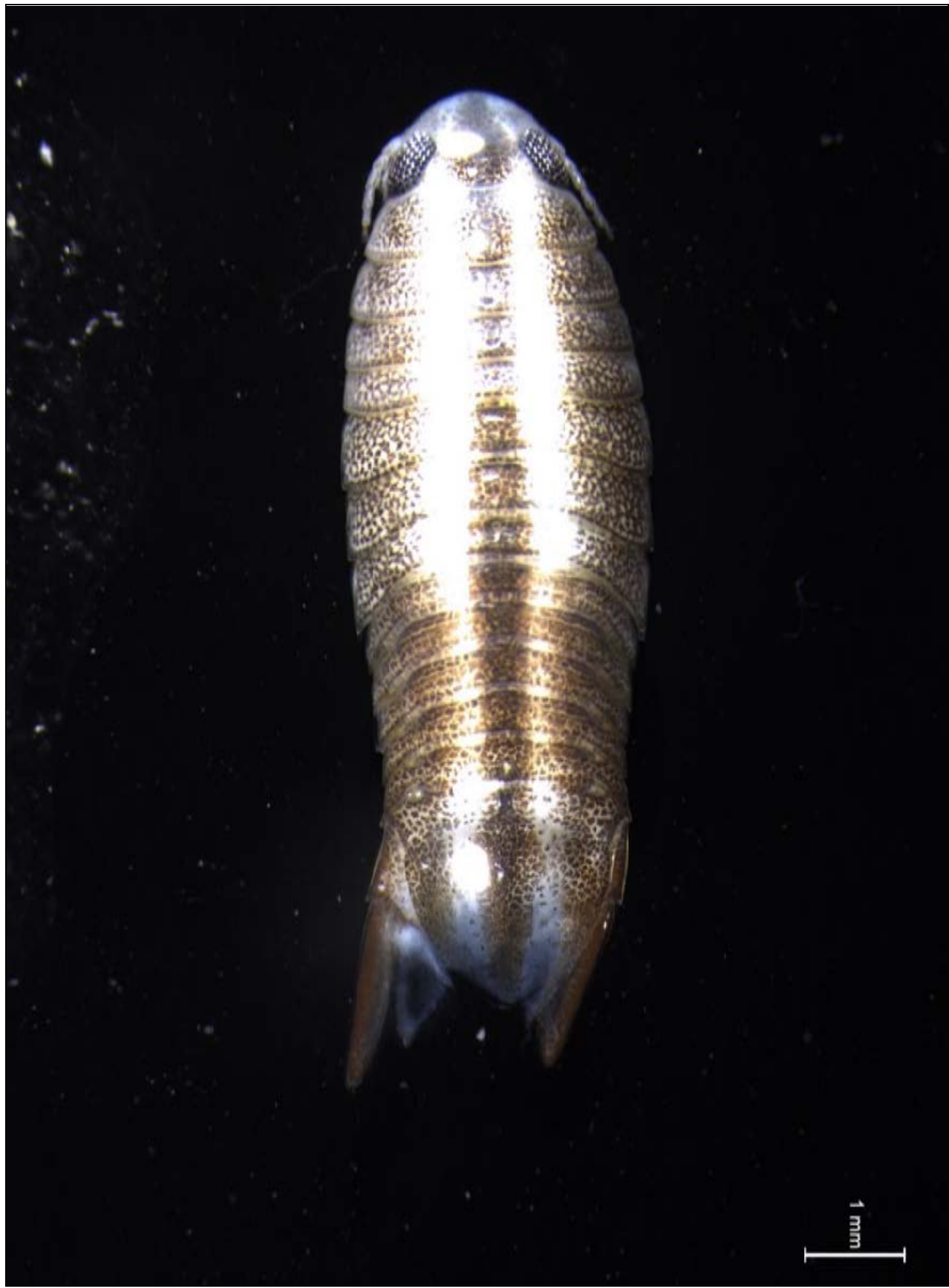

Figure 1: Nerocila orbignyi, male. 


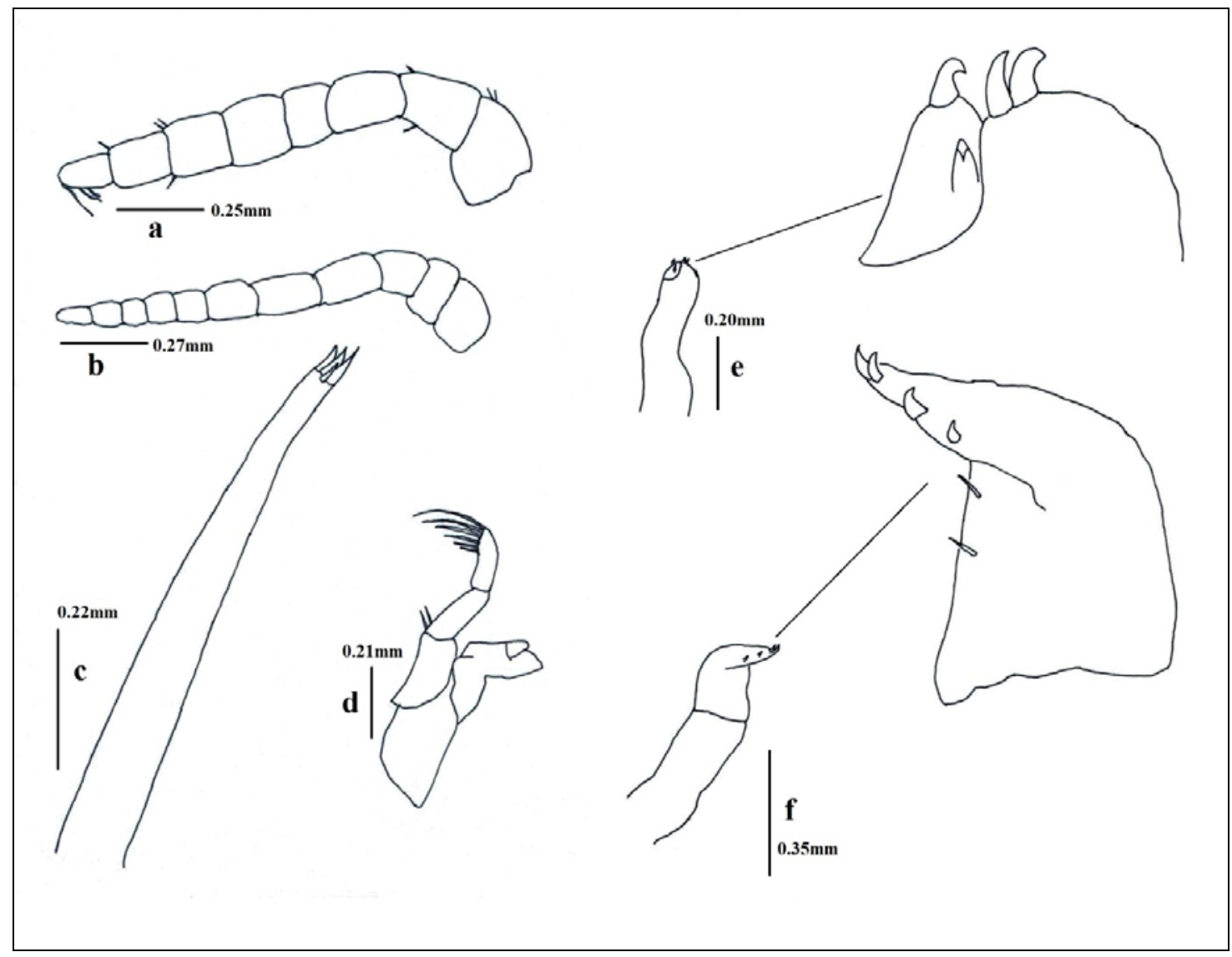

Figure 2: a) antennula, b) antenna, c) maxillula, d) mandible, e) maxilla, f) maxilliped.

Number of examined fish 109; number of infested fish eight; prevalence 7.33\%; mean intensity 1; infestation site on host on ventral and pectoral fins.

Male morphological characteristics: body length varies from 6.8 to $9.5 \mathrm{~mm}$. Cephalon 0.5 times longer than wide, visible from dorsal view. Body three times as long as greatest width. Pereon longest at pereonite 1 , shortest at pereonite 4 . Pereon widest at pereonite 5 , most narrow at pereonite 1 . Eyes big, facets distinct, 0.2 times width of head. Coxal plates of pereonites not visible in dorsal view. All pleonites visible in dorsal view. Pleon longest at pereonite 5 , shortest at pereonite 1 . Pleon widest at pereonite 1 , most narrow at pereonite 5 . Pleon 1 and 2 not overlapped by pereonite 7 . Pleotelson 0.85 times as long as anterior width. Pleotelson not wider than pereonite 7 and pleonite 5. Pleotelson longer than width. Antennula (Fig. 2a) comprising eight articles; extending to posterior margin of eye. Antenna (Fig. 2.b) comprising 11 articles, extending to anterior margin of pereonite 1 . Antenna sligthly longer than antennula. Mandibular process (Fig. 2d) without simple setae. Third article of mandible palp sligthly shorter than others, article 3 with six setae; article 2 with two setae on lateral margin. Maxillula (Fig. 2c) with four terminal setae, one long, one short, two setae similar in length. Maxilla (Fig. 2e) mesial lobe with two robust setae, lateral lobe with two recurved setae. Maxilliped (Fig. 2f) comprising three articles, article 3 with 4 recurved robust setae. 


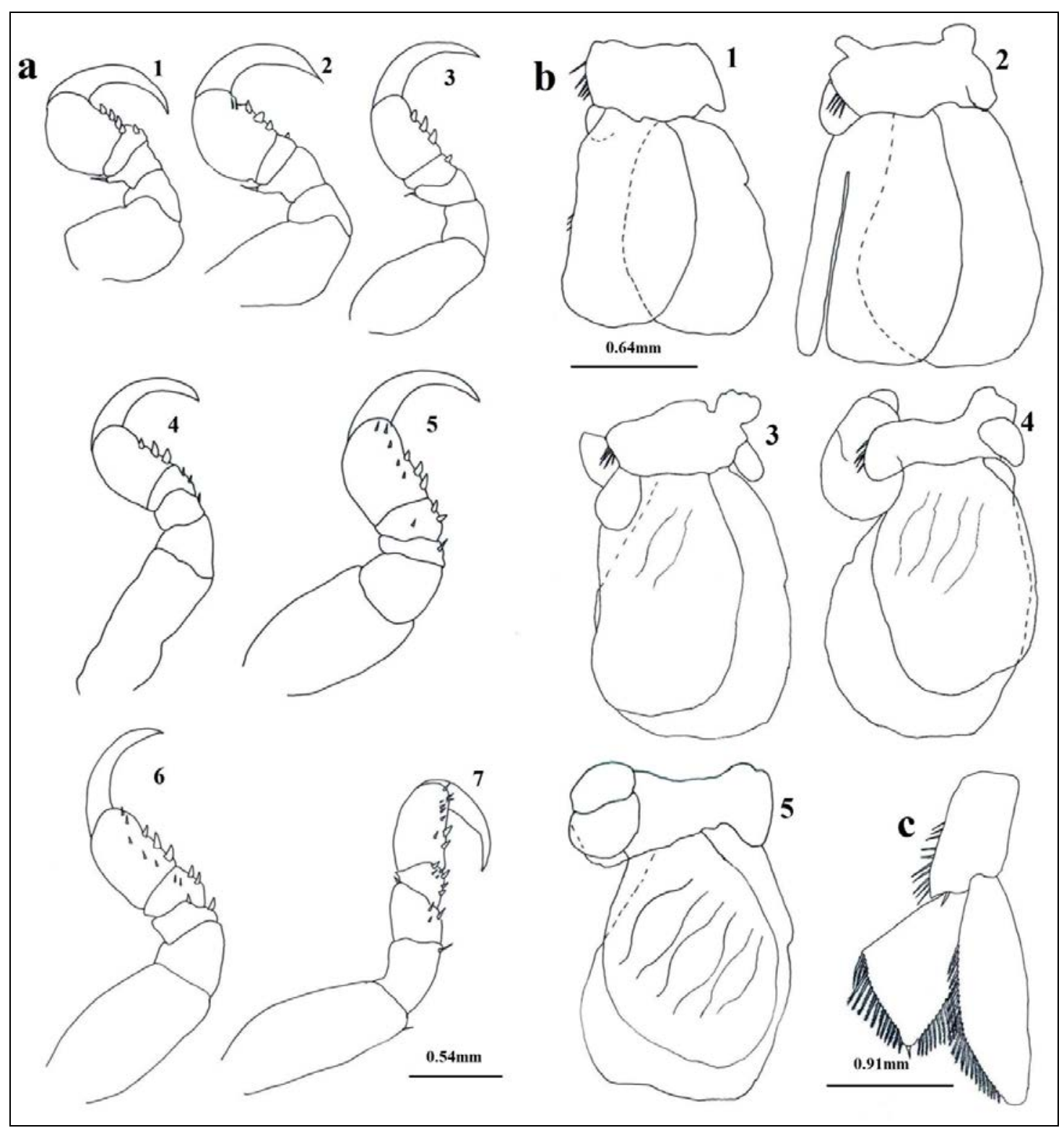

Figure 3: a) Pereopods 1-7, b) pleopods 1-5, c) uropod.

Pereopods 1-3 (Fig. 3a) similar in size, slightly smaller than pereopods 4-7. Three big spines on posterior margin of propodus of pereopods 2-6; four spines on posterior margin of propodus of pereopod 1; two spines on posterior margin of propodus of pereopods 7 . Besides, five spines on medial side of propodus of pereopods 5-6; eight spines with pereopod 7. One big spine on posterior margin of carpus of pereopods 1-3; two big spines on posterior margin of carpus of pereopods 4-6; five big spines posterior margin of carpus of pereopod 7. Small spines on medial side of carpus of pereopod 5-7. One spine of posterior margin of merus of pereopods 4-5; two spines posterior margin of merus of pereopods 6-7. Besides, one spine on anterior margin of merus of pereopods 1-3, 7. One spine on posterior margin of ischium and 
basis of pereopod 7. Pleopods (Fig. 3b) slightly decreasing in length. Pleopod 2 appendix masculina the same length as length of endopod. Peduncles of pleopods 1-4 with coupling hooks. Pleopod 1 exopod 1.4 times as long as wide, lateral and mesial margin slightly rounded, distally broadly rounded; endopod 1.6 times as long as wide, lateral margin staright, distally broadly rounded, mesial margin rounded; peduncle 1.8 times as wide as long. Exopod of uropod (Fig. 3c) longer than endopod; endopod extending beyond pleotelson posterior margin.

Eight genus belonging to the family Cymothoidae (Crustaceca, Isopoda) were reported from the Mediterranean such as Anilocra, Ceratothoa, Elthusa, Emetha, Idusa, Livoneca, Mothocya, and Nerocila (Trilles, 1997; Castello et al., 2020). Forty-two species were listed by The World Register of Marine Species (WoRMS, 2020) in the genus Nerocila. Four species (Nerocila acuminata, Nerocila bivittata, Nerocila orbignyi, and Nerocila milesensis) were reported from Turkish waters (Öktener and Trilles, 2004; Er and Kayış, 2015; Öktener et al., 2020).

Nerocila orbignyi is a parasite frequently reported from the body surfaces and fins of fish. It has been reported from North Atlantic Ocean, Mediterranean Sea, Adriatic Sea (Trilles 1994). It is associated with Actinopterygii and Elasmobranchii (Tab. 1).

Table 1: The hosts of Nerocila orbignyi with synonyms.

\begin{tabular}{|c|c|c|c|c|}
\hline$\tilde{z}$ & $\begin{array}{l}\text { Host fish species } \\
\text { (synonyms) }\end{array}$ & $\begin{array}{l}\text { Order, family of } \\
\text { host }\end{array}$ & Locality & References \\
\hline in & $\begin{array}{l}\text { Chimaera } \\
\text { sp. }\end{array}$ & $\begin{array}{l}\text { Chimaeriformes, } \\
\text { Chimaeridae. }\end{array}$ & $\begin{array}{l}\text { Warrnambool, } \\
\text { Victoria }\end{array}$ & Hale (1926) \\
\hline$\overbrace{\infty}^{\infty}$ & $\begin{array}{l}\text { Callorhinchus } \\
\text { milii }\end{array}$ & $\begin{array}{l}\text { Chimaeriformes, } \\
\text { Callorhinchidae. }\end{array}$ & $\begin{array}{l}\text { Tasmania, } \\
\text { Storm Bay }\end{array}$ & Hale (1940) \\
\hline 奈 & $\begin{array}{l}\text { Chelon auratus } \\
\text { (syn. } \\
\text { auratus) }\end{array}$ & $\begin{array}{l}\text { Mugiliformes, } \\
\text { Mugilidae. }\end{array}$ & $\begin{array}{l}\text { Thau and Vic- } \\
\text { Mireval ponds, and } \\
\text { Lake Patria in Italy }\end{array}$ & $\begin{array}{l}\text { Trilles }(1961,1962, \\
\text { 1964a, b, 1968) }\end{array}$ \\
\hline 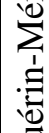 & Mugil cephalus & $\begin{array}{l}\text { Mugiliformes, } \\
\text { Mugilidae. }\end{array}$ & $\begin{array}{l}\text { Thau and Vic- } \\
\text { Mireval ponds, and } \\
\text { Lake Patria in Italy }\end{array}$ & $\begin{array}{l}\text { Trilles (1961, 1962, } \\
\text { 1964a, b, 1968) }\end{array}$ \\
\hline 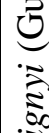 & $\begin{array}{l}\text { Chelon ramada } \\
\text { (syn. Mugil capito) }\end{array}$ & $\begin{array}{l}\text { Mugiliformes, } \\
\text { Mugilidae. }\end{array}$ & $\begin{array}{l}\text { Thau and Vic- } \\
\text { Mireval ponds, and } \\
\text { Lake Patria in Italy }\end{array}$ & $\begin{array}{l}\text { Trilles (1962, } \\
\text { 1964a, b, 1968) }\end{array}$ \\
\hline $\begin{array}{l}0 \\
0 \\
0 \\
0 \\
0 \\
0\end{array}$ & $\begin{array}{l}\text { Chelon labrosus } \\
\text { (syn. Mugil chelo) }\end{array}$ & $\begin{array}{l}\text { Mugiliformes, } \\
\text { Mugilidae. }\end{array}$ & $\begin{array}{l}\text { Thau and Vic- } \\
\text { Mireval ponds, and } \\
\text { Lake Patria in Italy }\end{array}$ & $\begin{array}{l}\text { Trilles (1962, } \\
\text { 1964a, b, 1968) }\end{array}$ \\
\hline $\bar{z}$ & $\begin{array}{l}\text { Dicentrarchus } \\
\text { labrax (syn. Labrax } \\
\text { lupus) }\end{array}$ & $\begin{array}{l}\text { Perciformes, } \\
\text { Moronidae. }\end{array}$ & $\begin{array}{l}\text { Thau and Vic- } \\
\text { Mireval ponds, and } \\
\text { Lake Patria in Italy }\end{array}$ & $\begin{array}{l}\text { Trilles (1962, } \\
\text { 1964a, b, 1968) }\end{array}$ \\
\hline
\end{tabular}


Table 1 (continued): The hosts of Nerocila orbignyi with synonyms.

\begin{tabular}{|c|c|c|c|c|}
\hline & $\begin{array}{l}\text { Host fish species } \\
\text { (svnonvms) }\end{array}$ & $\begin{array}{l}\text { Order, family of } \\
\text { host }\end{array}$ & Locality & References \\
\hline & $\begin{array}{l}\text { Platichthys flesus } \\
\text { (syn. Pleuronectes } \\
\text { flesus, Flesus } \\
\text { passer) }\end{array}$ & $\begin{array}{l}\text { Pleuronectiformes, } \\
\text { Pleuronectidae. }\end{array}$ & $\begin{array}{l}\text { Thau and Vic- } \\
\text { Mireval ponds, and } \\
\text { Lake Patria in Italy }\end{array}$ & $\begin{array}{l}\text { Trilles (1962, } \\
\text { 1964a, b, 1968) }\end{array}$ \\
\hline & $\begin{array}{l}\text { Oreochromis } \\
\text { spilurus (syn. } \\
\text { Tilapia galilea) }\end{array}$ & $\begin{array}{l}\text { Perciformes, } \\
\text { Cichlidae. }\end{array}$ & $\begin{array}{l}\text { Brackish lake in } \\
\text { Egypt }\end{array}$ & Wunder (1962) \\
\hline & Salmo trutta & $\begin{array}{l}\text { Salmoniformes, } \\
\text { Salmonidae. }\end{array}$ & - & Scott (1964) \\
\hline & Syphonostomes & - & - & Fain-Maurel (1966) \\
\hline 胥 & Mugilidae & $\begin{array}{l}\text { Mugiliformes, } \\
\text { Mugilidae. }\end{array}$ & - & Fain-Maurel (1966) \\
\hline 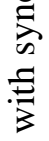 & $\begin{array}{l}\text { Argyrozona } \\
\text { argyrozona }\end{array}$ & $\begin{array}{l}\text { Perciformes, } \\
\text { Sparidae. }\end{array}$ & $\begin{array}{l}\text { Table, False, Algoa, } \\
\text { Senegal, North } \\
\text { Africa }\end{array}$ & Kensley (1978) \\
\hline 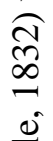 & \begin{tabular}{|l|} 
Pterogymnus \\
laniarius
\end{tabular} & $\begin{array}{l}\text { Perciformes, } \\
\text { Sparidae. }\end{array}$ & $\begin{array}{l}\text { Table, False, Algoa, } \\
\text { Senegal, North } \\
\text { Africa }\end{array}$ & Kensley (1978) \\
\hline 窎 & $\begin{array}{l}\text { Rhabdosargus } \\
\text { globiceps }\end{array}$ & $\begin{array}{l}\text { Perciformes, } \\
\text { Sparidae. }\end{array}$ & $\begin{array}{l}\text { Table, False, Algoa, } \\
\text { Senegal, North } \\
\text { Africa }\end{array}$ & Kensley (1978) \\
\hline 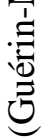 & Synaptura sp. & $\begin{array}{l}\text { Pleuronectiformes, } \\
\text { Soleidae. }\end{array}$ & $\begin{array}{l}\text { Table, False, Algoa, } \\
\text { Senegal, North } \\
\text { Africa }\end{array}$ & Kensley (1978) \\
\hline .ે & Thyrsites atun & $\begin{array}{l}\text { Perciformes, } \\
\text { Gempylidae. }\end{array}$ & $\begin{array}{l}\text { Table, False, Algoa, } \\
\text { Senegal, N. Africa }\end{array}$ & Kensley (1978) \\
\hline $\begin{array}{l}0 \\
0 \\
0 \\
0 \\
0\end{array}$ & $\begin{array}{l}\text { Chelon auratus } \\
\text { (syn. Mugil auratus) }\end{array}$ & $\begin{array}{l}\text { Mugiliformes, } \\
\text { Mugilidae. }\end{array}$ & Gulf of Marseille & Berner (1969) \\
\hline 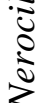 & $\begin{array}{l}\begin{array}{l}\text { Chelon ramada } \\
\text { (syn. Mugil capito) }\end{array} \\
\end{array}$ & $\begin{array}{l}\text { Mugiliformes, } \\
\text { Mugilidae. }\end{array}$ & Gulf of Marseille & Berner (1969) \\
\hline & $\begin{array}{l}\text { Chelon labrosus } \\
\text { (syn. Mugil chelo) }\end{array}$ & $\begin{array}{l}\text { Mugiliformes, } \\
\text { Mugilidae. }\end{array}$ & Gulf of Marseille & Berner (1969) \\
\hline & $\begin{array}{l}\text { Rhombosolea } \\
\text { plebeia }\end{array}$ & $\begin{array}{l}\text { Pleuronectiformes, } \\
\text { Rhombosoleidae. }\end{array}$ & $\begin{array}{l}\text { the Avon heathcote } \\
\text { estuary }\end{array}$ & Webb (1973) \\
\hline & $\begin{array}{l}\text { Rhombosolea } \\
\text { leporina }\end{array}$ & $\begin{array}{l}\text { Pleuronectiformes, } \\
\text { Rhombosoleidae. }\end{array}$ & $\begin{array}{l}\text { the Avon heathcote } \\
\text { estuary }\end{array}$ & Webb (1973) \\
\hline & $\begin{array}{l}\text { Chelon auratus } \\
\text { (syn. Mugil auratus) }\end{array}$ & $\begin{array}{l}\text { Mugiliformes, } \\
\text { Mugilidae. }\end{array}$ & Tunis Lake, Tunisia & $\begin{array}{l}\text { Trilles and Raibaut } \\
\text { (1973) }\end{array}$ \\
\hline & Mugil cephalus & $\begin{array}{l}\text { Mugiliformes, } \\
\text { Mugilidae. }\end{array}$ & Gulf of Tunis & $\begin{array}{l}\text { Trilles and Raibaut } \\
\text { (1973) }\end{array}$ \\
\hline & $\begin{array}{l}\text { Chelon ramada } \\
\text { (syn. Mugil capito) }\end{array}$ & $\begin{array}{l}\text { Mugiliformes, } \\
\text { Mugilidae. }\end{array}$ & $\begin{array}{l}\text { Ichkeul } \\
\text { Tunisia }\end{array}$ & $\begin{array}{l}\text { Trilles and Raibaut } \\
\text { (1973) }\end{array}$ \\
\hline
\end{tabular}


Table 1 (continued): The hosts of Nerocila orbignyi with synonyms.

\begin{tabular}{|c|c|c|c|c|}
\hline & Host fish species & Order, family of & Locality & References \\
\hline & 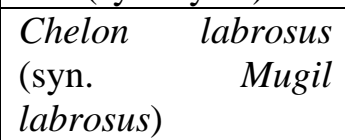 & $\begin{array}{l}\text { Mugiliformes, } \\
\text { Mugilidae. }\end{array}$ & Tunis Lake, Tunisia & $\begin{array}{l}\text { Trilles and Raibaut } \\
\text { (1973) }\end{array}$ \\
\hline & Alosa fallax & $\begin{array}{l}\text { Clupeiformes, } \\
\text { Clupeidae. }\end{array}$ & Tunisia & $\begin{array}{l}\text { Trilles and Raibaut } \\
\text { (1973) }\end{array}$ \\
\hline & $\begin{array}{ll}\text { Alosa fallax } & \text { (syn. } \\
\text { Alosa } & \text { fallax } \\
\text { nilotica) } & \\
\end{array}$ & $\begin{array}{l}\text { Clupeiformes, } \\
\text { Clupeidae. }\end{array}$ & Tunisia & $\begin{array}{l}\text { Trilles and Raibaut } \\
\text { (1973) }\end{array}$ \\
\hline 气 & \begin{tabular}{|lr}
$\begin{array}{l}\text { Chelon } \\
\text { (syn. }\end{array}$ & auratus \\
auratus) & Mugil \\
\end{tabular} & $\begin{array}{l}\text { Mugiliformes, } \\
\text { Mugilidae. }\end{array}$ & $\begin{array}{l}\text { Royan (lower } \\
\text { Charante), Gulf of } \\
\text { Gascogne }\end{array}$ & Trilles (1975) \\
\hline$\widehat{\Xi}$ & Mugil cephalus & $\begin{array}{l}\text { Mugiliformes, } \\
\text { Mugilidae. }\end{array}$ & $\begin{array}{l}\text { Royan (lower } \\
\text { Charante), Gulf of } \\
\text { Gascogne }\end{array}$ & Trilles (1975) \\
\hline$\frac{\sqrt[5]{3}}{3}$ & $\begin{array}{l}\text { Chelon ramada } \\
\text { (syn. Mugil capito) }\end{array}$ & $\begin{array}{l}\text { Mugiliformes, } \\
\text { Mugilidae. }\end{array}$ & $\begin{array}{l}\text { Royan (lower } \\
\text { Charante), Gulf of } \\
\text { Gascogne }\end{array}$ & Trilles (1975) \\
\hline$\stackrel{\leftrightarrow}{=}$ & $\begin{array}{l}\text { Chelon labrosus } \\
\text { (syn. Mugil chelo) }\end{array}$ & $\begin{array}{l}\text { Mugiliformes, } \\
\text { Mugilidae. }\end{array}$ & $\begin{array}{l}\text { Royan (Charante), } \\
\text { Gulf of Gascogne }\end{array}$ & Trilles (1975) \\
\hline$\sum_{i=1}^{\infty}$ & $\begin{array}{l}\text { Platichthys flesus } \\
\text { (syn. Pleuronectes } \\
\text { flesus, Flesus } \\
\text { passer) }\end{array}$ & $\begin{array}{l}\text { Pleuronectiformes, } \\
\text { Pleuronectidae. }\end{array}$ & $\begin{array}{l}\text { Royan (lower } \\
\text { Charante), Gulf of } \\
\text { Gascogne }\end{array}$ & Trilles (1975) \\
\hline تُ & $\begin{array}{l}\text { Dicentrarchus } \\
\text { labrax (syn. Labrax } \\
\text { lupus) }\end{array}$ & $\begin{array}{l}\text { Perciformes, } \\
\text { Moronidae. }\end{array}$ & $\begin{array}{l}\text { Royan (lower } \\
\text { Charante), Gulf of } \\
\text { Gascogne }\end{array}$ & Trilles (1975) \\
\hline $\begin{array}{l}0 \\
0 \\
0 \\
0 \\
0\end{array}$ & $\begin{array}{l}\text { Halobatrachus } \\
\text { didactylus (syn. } \\
\text { Batrachoides } \\
\text { didactylus) }\end{array}$ & $\begin{array}{l}\text { Batrachoidiformes, } \\
\text { Batrachoididae. }\end{array}$ & $\begin{array}{l}\text { Temara-isthme, } \\
\text { Rabat, Morocco }\end{array}$ & $\begin{array}{l}\text { Dollfus and Trilles } \\
\text { (1976) }\end{array}$ \\
\hline z & Solea senegalensis & $\begin{array}{l}\text { Pleuronectiformes, } \\
\text { Soleidae. }\end{array}$ & Rabat, Morocco & $\begin{array}{l}\text { Dollfus and Trilles } \\
\text { (1976) }\end{array}$ \\
\hline & $\begin{array}{l}\text { Dicentrarchus } \\
\text { labrax }\end{array}$ & $\begin{array}{l}\text { Perciformes, } \\
\text { Moronidae. }\end{array}$ & $\begin{array}{l}\text { the pond Diana } \\
\text { (Corsica) }\end{array}$ & $\begin{array}{l}\text { Bragoni et } \\
(1983)\end{array}$ al. \\
\hline & $\begin{array}{l}\text { Dicentrarchus } \\
\text { labrax }\end{array}$ & $\begin{array}{l}\text { Perciformes, } \\
\text { Moronidae. }\end{array}$ & $\begin{array}{l}\text { the pond Diana } \\
\text { (Corsica) }\end{array}$ & $\begin{array}{l}\text { Bragoni et } \\
(1984)\end{array}$ al. \\
\hline & $\begin{array}{l}\text { Pleuronectidae and } \\
\text { Soleidae }\end{array}$ & Pleuronectiformes. & & Rokicki (1985) \\
\hline & $\begin{array}{l}\text { Chelon saliens (syn. } \\
\text { Liza saliens) }\end{array}$ & $\begin{array}{l}\text { Mugiliformes, } \\
\text { Mugilidae. }\end{array}$ & Montenegro & Trilles et al. (1989) \\
\hline & $\begin{array}{l}\text { Chelon auratus } \\
\text { (syn. Liza aurata) }\end{array}$ & $\begin{array}{l}\text { Mugiliformes, } \\
\text { Mugilidae. }\end{array}$ & Montenegro & Trilles et al. (1989) \\
\hline
\end{tabular}


Table 1 (continued): The hosts of Nerocila orbignyi with synonyms.

\begin{tabular}{|c|c|c|c|c|}
\hline & Host fish species & Order, family of & Locality & References \\
\hline & $\begin{array}{l}\text { Chelon ramada } \\
\text { (syn. Liza ramada) }\end{array}$ & $\begin{array}{l}\text { Mugiliformes, } \\
\text { Mugilidae. }\end{array}$ & Montenegro & Trilles et al. (1989) \\
\hline & Chelon labrosus & $\begin{array}{l}\text { Mugiliformes, } \\
\text { Mugilidae. }\end{array}$ & Montenegro & Trilles et al. (1989) \\
\hline & Lophius budegassa & $\begin{array}{l}\text { Lophiiformes, } \\
\text { Lophiidae. }\end{array}$ & Montenegro & Trilles et al. (1989) \\
\hline & Belone belone & $\begin{array}{l}\text { Beloniformes, } \\
\text { Belonidae. }\end{array}$ & $\begin{array}{l}\text { Courtmascsherry } \\
\text { Bay }\end{array}$ & $\begin{array}{ll}\text { Dorman } & \text { and } \\
\text { Holmes (1991) }\end{array}$ \\
\hline & Prionotus sp. & $\begin{array}{l}\text { Scorpaeniformes, } \\
\text { Triglidae. }\end{array}$ & Amazonas, Brazil & Thatcher (1995) \\
\hline 气 & $\begin{array}{l}\text { Merlangius } \\
\text { merlangus }\end{array}$ & $\begin{array}{l}\text { Gadiformes, } \\
\text { Gadidae. }\end{array}$ & $\begin{array}{l}\text { The Severn Estuary, } \\
\text { Bristol Channel }\end{array}$ & $\begin{array}{l}\text { Potter et al. (1989) } \\
\text { (1988) }\end{array}$ \\
\hline & $\begin{array}{l}\text { Chelon ramada } \\
\text { (syn. Liza ramada) }\end{array}$ & $\begin{array}{l}\text { Mugiliformes, } \\
\text { Mugilidae. }\end{array}$ & $\begin{array}{l}\text { Ghar El Melh, } \\
\text { Tunisia }\end{array}$ & $\begin{array}{l}\text { Charfi-Cheikhrouha } \\
\text { et al. (2000) }\end{array}$ \\
\hline & $\begin{array}{l}\text { Chelon saliens (syn. } \\
\text { Liza saliens) }\end{array}$ & $\begin{array}{l}\text { Mugiliformes, } \\
\text { Mugilidae. }\end{array}$ & Bizerte, Tunisia & $\begin{array}{l}\text { Charfi-Cheikhrouha } \\
\text { et al. (2000) }\end{array}$ \\
\hline & $\begin{array}{l}\text { Chelon auratus } \\
\text { (syn. Liza aurata) }\end{array}$ & $\begin{array}{l}\text { Mugiliformes, } \\
\text { Mugilidae. }\end{array}$ & $\begin{array}{l}\text { Ghar El Melh, } \\
\text { Tunisia }\end{array}$ & $\begin{array}{l}\text { Charfi-Cheikhrouha } \\
\text { et al. (2000) }\end{array}$ \\
\hline & Chelon labrosus & $\begin{array}{l}\text { Mugiliformes, } \\
\text { Mugilidae. }\end{array}$ & Sfax, Tunisia & $\begin{array}{l}\text { Charfi-Cheikhrouha } \\
\text { et al. (2000) }\end{array}$ \\
\hline & $\begin{array}{l}\text { Dicentrarchus } \\
\text { labrax }\end{array}$ & $\begin{array}{l}\text { Perciformes, } \\
\text { Moronidae. }\end{array}$ & $\begin{array}{l}\text { Ghar El Melh, } \\
\text { Tunisia }\end{array}$ & $\begin{array}{l}\text { Charfi-Cheikhrouha } \\
\text { et al. (2000) }\end{array}$ \\
\hline & Solea solea & $\begin{array}{l}\text { Pleuronectiformes, } \\
\text { Soleidae. }\end{array}$ & Sfax, Tunisia & $\begin{array}{l}\text { Charfi-Cheikhrouha } \\
\text { et al. (2000) }\end{array}$ \\
\hline 5 & Serranus scriba & $\begin{array}{l}\text { Perciformes, } \\
\text { Serranidae. }\end{array}$ & Sfax, Tunisia & $\begin{array}{l}\text { Charfi-Cheikhrouha } \\
\text { et al. (2000) }\end{array}$ \\
\hline & Diplodus annularis & $\begin{array}{l}\text { Perciformes, } \\
\text { Sparidae. }\end{array}$ & Sfax, Tunisia & $\begin{array}{l}\text { Charfi-Cheikhrouha } \\
\text { et al. (2000) }\end{array}$ \\
\hline & Arripis trutta & $\begin{array}{l}\text { Perciformes, } \\
\text { Arripidae. }\end{array}$ & New Zealand & Hine et al. (2000) \\
\hline & $\begin{array}{l}\text { Dicentrarchus } \\
\text { labrax }\end{array}$ & $\begin{array}{l}\text { Perciformes, } \\
\text { Moronidae. }\end{array}$ & Aegean Sea, Turkey & $\begin{array}{l}\text { Horton and } \\
\text { Okamura (2001) }\end{array}$ \\
\hline & $\begin{array}{l}\text { Chelon auratus } \\
\text { (syn. Liza aurata) }\end{array}$ & $\begin{array}{l}\text { Mugiliformes, } \\
\text { Mugilidae. }\end{array}$ & $\begin{array}{ll}\text { Mistras } & \text { Lagoon, } \\
\text { Sardinia } & \end{array}$ & $\begin{array}{l}\text { Merella and } \\
\text { Garippa (2001) }\end{array}$ \\
\hline & Seriola dumerili & $\begin{array}{l}\text { Perciformes, } \\
\text { Carangidae. }\end{array}$ & Mazarrón (Murcia) & Montero (2001) \\
\hline & $\begin{array}{l}\text { Chelon auratus } \\
\text { (syn. Liza aurata) }\end{array}$ & $\begin{array}{l}\text { Mugiliformes, } \\
\text { Mugilidae. }\end{array}$ & Black Sea & $\begin{array}{l}\text { Öktener and Trilles } \\
\text { (2004) }\end{array}$ \\
\hline & $\begin{array}{l}\text { Halobatrachus } \\
\text { didactylus }\end{array}$ & $\begin{array}{l}\text { Batrachoidiformes, } \\
\text { Batrachoididae. }\end{array}$ & $\begin{array}{l}\text { Portuguese coast } \\
\text { Guadiana, Tagus, } \\
\text { Sado, Olha o and } \\
\text { Tavira coastal areas }\end{array}$ & $\begin{array}{l}\text { Marques et al. } \\
(2005)\end{array}$ \\
\hline
\end{tabular}


Table 1 (continued): The hosts of Nerocila orbignyi with synonyms.

\begin{tabular}{|c|c|c|c|c|}
\hline & $\begin{array}{l}\text { Host fish species } \\
\text { (synonyms) }\end{array}$ & $\begin{array}{l}\text { Order, family of } \\
\text { host }\end{array}$ & Locality & References \\
\hline & Mugil cephalus & $\begin{array}{l}\text { Mugiliformes, } \\
\text { Mugilidae. }\end{array}$ & $\begin{array}{l}\text { Béjaïa, Soummam } \\
\text { Oued, Algeria }\end{array}$ & $\begin{array}{l}\text { Ramdane et al. } \\
\text { (2007) }\end{array}$ \\
\hline & $\begin{array}{l}\text { Symphodus tinca } \\
\text { (Crenilabrus pavo) }\end{array}$ & $\begin{array}{l}\text { Perciformes, } \\
\text { Labridae. }\end{array}$ & $\begin{array}{l}\text { Gulf of Béjaïa, } \\
\text { Algeria }\end{array}$ & $\begin{array}{l}\text { Ramdane et al. } \\
\text { (2007) }\end{array}$ \\
\hline & $\begin{array}{l}\text { Symphodus tinca } \\
\text { (Crenilabrus pavo) }\end{array}$ & $\begin{array}{l}\text { Perciformes, } \\
\text { Labridae. }\end{array}$ & $\begin{array}{l}\text { Gulf of } \\
\text { Algeria }\end{array}$ & $\begin{array}{l}\text { Ramdane et al. } \\
\text { (2007) }\end{array}$ \\
\hline & Trigla lyra & $\begin{array}{l}\text { Scorpaeniformes, } \\
\text { Triglidae. }\end{array}$ & $\begin{array}{l}\text { Gulf of Béjaïa, } \\
\text { Algeria }\end{array}$ & $\begin{array}{l}\text { Ramdane et al. } \\
(2007)\end{array}$ \\
\hline & Platichthys flesus & $\begin{array}{l}\text { Pleuronectiformes, } \\
\text { Pleuronectidae. }\end{array}$ & $\begin{array}{l}\text { North-Central } \\
\text { Portuguese Coast }\end{array}$ & $\begin{array}{ll}\text { Cavaleiro } & \text { and } \\
\text { Santos (2007) } & \\
\end{array}$ \\
\hline$\tilde{\Xi}$ & Scorpaena porcus & $\begin{array}{l}\text { Scorpaeniformes, } \\
\text { Scorpaenidae. }\end{array}$ & Adriatic Sea & Ferri et al. (2008) \\
\hline 胥 & $\begin{array}{l}\text { Monochirus } \\
\text { hispidus }\end{array}$ & $\begin{array}{l}\text { Pleuronectiformes, } \\
\text { Soleidae. }\end{array}$ & Northeast Atlantic & $\begin{array}{l}\text { Marques et } \\
\text { (2009) }\end{array}$ \\
\hline $\begin{array}{c}5 \\
\frac{7}{2} \\
\frac{2}{3}\end{array}$ & Solea solea & $\begin{array}{l}\text { Pleuronectiformes, } \\
\text { Soleidae. }\end{array}$ & $\begin{array}{lr}\text { Pazar } \quad \text { Coast, } \\
\text { Turkey, Black Sea }\end{array}$ & $\begin{array}{l}\text { Kayiş and Ceylan } \\
\text { (2011) }\end{array}$ \\
\hline$\underset{\infty}{\stackrel{3}{a}}$ & $\begin{array}{l}\text { Chelidonichthys } \\
\text { capensis }\end{array}$ & $\begin{array}{l}\text { Scorpaeniformes, } \\
\text { Triglidae. }\end{array}$ & South Africa & $\begin{array}{l}\begin{array}{l}\text { Hadfield et al. } \\
(2013)\end{array} \\
\end{array}$ \\
\hline 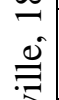 & Moolgarda seheli & $\begin{array}{l}\text { Mugiliformes, } \\
\text { Mugilidae. }\end{array}$ & Red Sea, Yemen & $\begin{array}{l}\text { Al-Zubaidy and } \\
\text { Mhaisen (2013) }\end{array}$ \\
\hline 总 & $\begin{array}{l}\text { Chelon auratus } \\
\text { (syn. Liza aurata) }\end{array}$ & $\begin{array}{l}\text { Mugiliformes, } \\
\text { Mugilidae. }\end{array}$ & Red Sea, Yemen & $\begin{array}{l}\text { Al-Zubaidy and } \\
\text { Mhaisen (2013) }\end{array}$ \\
\hline 紊 & Serranus cabrilla & $\begin{array}{l}\text { Perciformes, } \\
\text { Serranidae. }\end{array}$ & $\begin{array}{l}\text { Samandağ, Turkey, } \\
\text { Mediterranean }\end{array}$ & Özcan et al. (2015) \\
\hline$\underbrace{0}_{-\pi}$ & $\begin{array}{l}\text { Belone belone (syn. } \\
\text { Belone b. gracilis) }\end{array}$ & $\begin{array}{l}\text { Beloniformes, } \\
\text { Belonidae. }\end{array}$ & Tunisia & Châari et al. (2015) \\
\hline 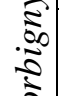 & $\begin{array}{l}\text { Chelon ramada } \\
\text { (syn. Mugil capito) }\end{array}$ & $\begin{array}{l}\text { Mugiliformes, } \\
\text { Mugilidae. }\end{array}$ & Qaroun Lake, Egypt & $\begin{array}{l}\text { Shaheen et al. } \\
\text { (2017) }\end{array}$ \\
\hline $\mid \begin{array}{c}0 \\
0 \\
\\
\end{array}$ & Solea solea & $\begin{array}{l}\text { Pleuronectiformes, } \\
\text { Soleidae. }\end{array}$ & Qaroun Lake, Egypt & $\begin{array}{lll}\begin{array}{l}\text { Shaheen et } \\
(2017)\end{array} & \text { al. }\end{array}$ \\
\hline 京 & $\begin{array}{l}\text { Coptodon zillii (syn. } \\
\text { Tilapia zilli) }\end{array}$ & $\begin{array}{l}\text { Perciformes, } \\
\text { Cichlidae. }\end{array}$ & Qaroun Lake, Egypt & $\begin{array}{l}\begin{array}{l}\text { Shaheen et al. } \\
(2017)\end{array} \\
\left(\begin{array}{l} \\
(20)\end{array}\right.\end{array}$ \\
\hline & $\begin{array}{l}\text { Chelon ramada } \\
\text { (syn. Mugil capito) }\end{array}$ & $\begin{array}{l}\text { Mugiliformes, } \\
\text { Mugilidae. }\end{array}$ & Qaroun Lake, Egypt & $\begin{array}{l}\text { Mahmoud et al. } \\
(2017)\end{array}$. \\
\hline & Liza carinata & $\begin{array}{l}\text { Mugiliformes, } \\
\text { Mugilidae. }\end{array}$ & Qaroun Lake, Egypt & $\begin{array}{l}\text { Mahmoud et al. } \\
(2017)\end{array}$ \\
\hline & $\begin{array}{l}\text { Coptodon zillii (syn. } \\
\text { Tilapia zilli) }\end{array}$ & $\begin{array}{l}\text { Perciformes, } \\
\text { Cichlidae. }\end{array}$ & Qaroun Lake, Egypt & $\begin{array}{l}\text { Mahmoud et al. } \\
(2017)\end{array}$ \\
\hline & Scomber scombrus & $\begin{array}{l}\text { Perciformes, } \\
\text { Scombridae. }\end{array}$ & $\begin{array}{l}\text { Swanage, } \\
\text { waters }\end{array}$ & $\begin{array}{l}\text { Horton and Baillie } \\
\text { (2019) }\end{array}$ \\
\hline & Clupea harengus & $\begin{array}{l}\text { Clupeiformes, } \\
\text { Clupeidae. }\end{array}$ & $\begin{array}{l}\text { Southampton, UK } \\
\text { waters }\end{array}$ & $\begin{array}{l}\text { Horton and Baillie } \\
\text { (2019) }\end{array}$ \\
\hline & Chelon labrosus & $\begin{array}{l}\text { Mugiliformes, } \\
\text { Mugilidae. }\end{array}$ & $\begin{array}{l}\text { Newlyn harbour, } \\
\text { UK waters }\end{array}$ & $\begin{array}{l}\text { Horton and Baillie } \\
\text { (2019) }\end{array}$ \\
\hline
\end{tabular}


Table 1 (continued): The hosts of Nerocila orbignyi with synonyms.

\begin{tabular}{|c|c|c|c|c|}
\hline & $\begin{array}{l}\text { Host fish species } \\
\text { (synonyms) }\end{array}$ & $\begin{array}{c}\text { Order, family of } \\
\text { host }\end{array}$ & Locality & References \\
\hline & Solea solea & $\begin{array}{l}\text { Pleuronectiformes, } \\
\text { Soleidae. }\end{array}$ & $\begin{array}{ll}\text { Sinop, } & \text { Turkey, } \\
\text { Black Sea } & \end{array}$ & $\begin{array}{l}\text { Güven and Öztürk } \\
\text { (2019) }\end{array}$ \\
\hline & Salmo salar & $\begin{array}{l}\text { Salmoniformes, } \\
\text { Salmonidae. }\end{array}$ & Tasmania, Australia & $\begin{array}{l}\begin{array}{l}\text { González et al. } \\
\text { (2019) }\end{array} \\
\text { (2) }\end{array}$ \\
\hline & Callorhynchus milii & $\begin{array}{l}\text { Chimaeriformes, } \\
\text { Callorhinchidae. }\end{array}$ & $\begin{array}{lr}\text { Bass } & \text { Strait, } \\
\text { Nutgrove } \quad \text { Beach, } \\
\text { Derwent Estuary }\end{array}$ & Bruce (1987) \\
\hline & $\begin{array}{l}\text { Pseudocaranx } \\
\text { dentex }\end{array}$ & $\begin{array}{l}\text { Perciformes, } \\
\text { Carangidae. }\end{array}$ & $\begin{array}{l}\text { Southern Western } \\
\text { Australia, Walpole }\end{array}$ & Bruce (1987) \\
\hline & $\begin{array}{l}\text { Dactyloptena } \\
\text { orientalis }\end{array}$ & $\begin{array}{l}\text { Scorpaeniformes, } \\
\text { Dactylopteridae. }\end{array}$ & $\begin{array}{ll}\text { South } & \text { Coast, } \\
\text { Tasmania } & \\
\end{array}$ & Bruce (1987) \\
\hline & Girella tricuspidata & $\begin{array}{l}\text { Perciformes, } \\
\text { Girellidae. }\end{array}$ & $\begin{array}{l}\text { Lake Illawarra, } \\
\text { New South Wales, } \\
\text { Australia }\end{array}$ & Bruce (1987) \\
\hline & Mola mola & $\begin{array}{l}\text { Tetraodontiforms, } \\
\text { Molidae. }\end{array}$ & $\begin{array}{l}\text { Off New South } \\
\text { Wales, Australia }\end{array}$ & Bruce (1987) \\
\hline & Mugillidae sp. & $\begin{array}{l}\text { Mugiliformes, } \\
\text { Mugilidae. }\end{array}$ & $\begin{array}{l}\text { Port Hacking, New } \\
\text { S. Wales, Australia }\end{array}$ & Bruce (1987) \\
\hline & Platycephalidae sp. & $\begin{array}{l}\text { Scorpaeniformes, } \\
\text { Platycephalidae. }\end{array}$ & Victoria & Bruce (1987) \\
\hline & $\begin{array}{l}\text { Pomatomus } \\
\text { saltatrix }\end{array}$ & $\begin{array}{l}\text { Perciformes, } \\
\text { Pomatomidae. }\end{array}$ & $\begin{array}{l}\text { Nornalup, Western } \\
\text { Australia }\end{array}$ & Bruce (1987) \\
\hline & Sillago bassensis & $\begin{array}{l}\text { Perciformes, } \\
\text { Sillaginidae. }\end{array}$ & $\begin{array}{l}\text { FrederickHenryBay, } \\
\text { Storm Bay, Tasmania }\end{array}$ & Bruce (1987) \\
\hline & $\begin{array}{l}\text { Acanthopagrus } \\
\text { butcheri }\end{array}$ & $\begin{array}{l}\text { Perciformes, } \\
\text { Sparidae. }\end{array}$ & $\begin{array}{l}\text { The Coorong, South } \\
\text { Australia }\end{array}$ & Bruce (1987) \\
\hline 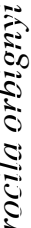 & $\begin{array}{l}\text { Pagrus auratus } \\
\text { (syn. Chrysophrys } \\
\text { auratus, } \\
\text { Pagrosomus } \\
\text { auritus) }\end{array}$ & $\begin{array}{l}\text { Perciformes, } \\
\text { Sparidae. }\end{array}$ & $\begin{array}{l}\text { Sydney, New South } \\
\text { Wales, Palm Beach, } \\
\text { Broken Bay, Little } \\
\text { Jerusalem Bay, } \\
\text { Hawkesbury River }\end{array}$ & Bruce (1987) \\
\hline$\sum$ & $\begin{array}{l}\text { Chelidonichthys } \\
\text { kumu }\end{array}$ & $\begin{array}{l}\text { Scorpaeniformes, } \\
\text { Triglidae. }\end{array}$ & $\begin{array}{l}\text { Southern Western } \\
\text { Australia, Walpole }\end{array}$ & Bruce (1987) \\
\hline & Mugil cephalus & $\begin{array}{l}\text { Mugiliformes, } \\
\text { Mugilidae. }\end{array}$ & Israel & $\begin{array}{l}\text { Paperna and } \\
\text { Overstreet (1981) }\end{array}$ \\
\hline & $\begin{array}{l}\text { Dicentrarchus } \\
\text { labrax }\end{array}$ & $\begin{array}{l}\text { Perciformes, } \\
\text { Moronidae. }\end{array}$ & Black Sea & Kayış et al. (2017) \\
\hline & $\begin{array}{l}\text { Chelon auratus } \\
\text { (syn. Liza aurata) }\end{array}$ & $\begin{array}{l}\text { Mugiliformes, } \\
\text { Mugilidae. }\end{array}$ & Black Sea & Kayış et al. (2017) \\
\hline & Serranus cabrilla & $\begin{array}{l}\text { Perciformes, } \\
\text { Serranidae. }\end{array}$ & Black Sea & Kayış et al. (2017) \\
\hline & Solea solea & $\begin{array}{l}\text { Pleuronectiformes, } \\
\text { Soleidae. }\end{array}$ & Black Sea & Kayış et al. (2017) \\
\hline
\end{tabular}


Table 1 (continued): The hosts of Nerocila orbignyi with synonyms.

\begin{tabular}{|c|c|c|c|c|}
\hline & $\begin{array}{l}\text { Host Fish Species } \\
\text { (synonyms) }\end{array}$ & $\begin{array}{c}\text { Order, Family of } \\
\text { Host }\end{array}$ & Locality & References \\
\hline & Unknown & Unknown & Castellon, Spain & Balcells (1953) \\
\hline & Raja sp. & Rajiformes, Rajidae & $\begin{array}{l}\text { the Croisic vicinity, } \\
\text { France }\end{array}$ & Trilles (1975) \\
\hline 윙 & $\begin{array}{l}\text { Trisopterus } \\
\text { capelanus (syn. } \\
\text { Gadus capelanus) }\end{array}$ & $\begin{array}{l}\text { Gadiformes, } \\
\text { Gadidae }\end{array}$ & Algeria & $\begin{array}{l}\text { Dollfus and Trilles } \\
\text { (1976) }\end{array}$ \\
\hline t. & Unknown & Unknown & $\begin{array}{l}\text { Province de Bou } \\
\text { Haroun, Algeria }\end{array}$ & $\begin{array}{l}\text { Dollfus and Trilles } \\
\text { (1976) }\end{array}$ \\
\hline 第 & $\begin{array}{l}\text { Rostroraja alba } \\
\text { (syn. Raja alba) }\end{array}$ & Rajiformes, Rajidae & $\begin{array}{l}\text { Casablanca, } \\
\text { Morocco }\end{array}$ & $\begin{array}{l}\text { Dollfus and Trilles } \\
\text { (1976) }\end{array}$ \\
\hline : & Unknown & Unknown & $\begin{array}{l}\text { Turkey, Spain, } \\
\text { Yougoslavie }\end{array}$ & Trilles (1977) \\
\hline = & Raja clavata & Rajiformes, Rajidae & Yougoslavie & Trilles (1977) \\
\hline 离 & $\begin{array}{l}\text { Chelon labrosus } \\
\text { (syn. Crenimugil } \\
\text { labrosus) }\end{array}$ & $\begin{array}{l}\text { Mugiliformes, } \\
\text { Mugilidae }\end{array}$ & The Island of Texel & Trilles (1977) \\
\hline ह & Unknown & Unknown & Naples, Genova & Trilles (1977) \\
\hline 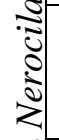 & $\begin{array}{lr}\begin{array}{l}\text { Chelon } \\
\text { (syn. }\end{array} \quad \text { labrosus } \\
\text { labrosus) }\end{array}$ & $\begin{array}{l}\text { Mugiliformes, } \\
\text { Mugilidae }\end{array}$ & Wadden Sea & Holthuis (1978) \\
\hline 常 & $\begin{array}{l}\begin{array}{l}\text { Chelon } \\
\text { (syn. }\end{array} \quad \text { Crenimugil } \\
\text { labrosus) }\end{array}$ & $\begin{array}{l}\text { Mugiliformes, } \\
\text { Mugilidae }\end{array}$ & The Island of Texel & $\begin{array}{l}\text { Adema and Huwae } \\
\text { (1982) }\end{array}$ \\
\hline क & Trisopterus minutus & $\begin{array}{l}\text { Gadiformes, } \\
\text { Gadidae }\end{array}$ & Montenegro & Trilles et al. (1989) \\
\hline & Trachinus draco & $\begin{array}{l}\text { Perciformes, } \\
\text { Trachinidae }\end{array}$ & Montenegro & Trilles et al. (1989) \\
\hline & Pagellus acarne & $\begin{array}{l}\text { Perciformes, } \\
\text { Sparidae }\end{array}$ & $\begin{array}{l}\text { Gulf of Béjaïa, } \\
\text { Algeria }\end{array}$ & 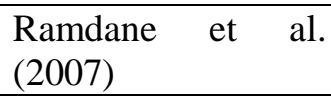 \\
\hline
\end{tabular}

The host fish of Nerocila orbignyi are examined according to their order; 22 (38\%) of 58 host species belong to Perciformes; 14 species (14\%) to Mugiliformes and Pleuronectiformes; 22 species (38\%) to different fish families (Fig. 4).

According to their families, the parasite appears to be more frequently reported from two families; Mugilidae and Sparidae with seven species (Fig. 5).

According to habitat selections; 24 (42\%) of 58 host fish species are demersal; 15 species (26\%) are benthopelagic, seven species (12\%) are pelagic-neritic, six species (10\%) are reef-associated, three species (5\%) are bathydemersal, three species (5\%) are pelagic-oceanic (Fig. 6). It seems that Nerocila orbignyi clearly prefers demersal fish than pelagic fish. Luque et al. (2010) and Brusca (1981) verified the occurrence of higher parasite species on demersal fishes in their studies.

According to feeding habits; 44 species (76\%) of 58 host fish species are carnivorous; 12 species (21\%) are omnivorous; two species (3\%) are herbivorous (Fig. 7). Nerocila orbignyi appears to prefer carnivorous fish rather than omnivorous and herbivorous fish. 


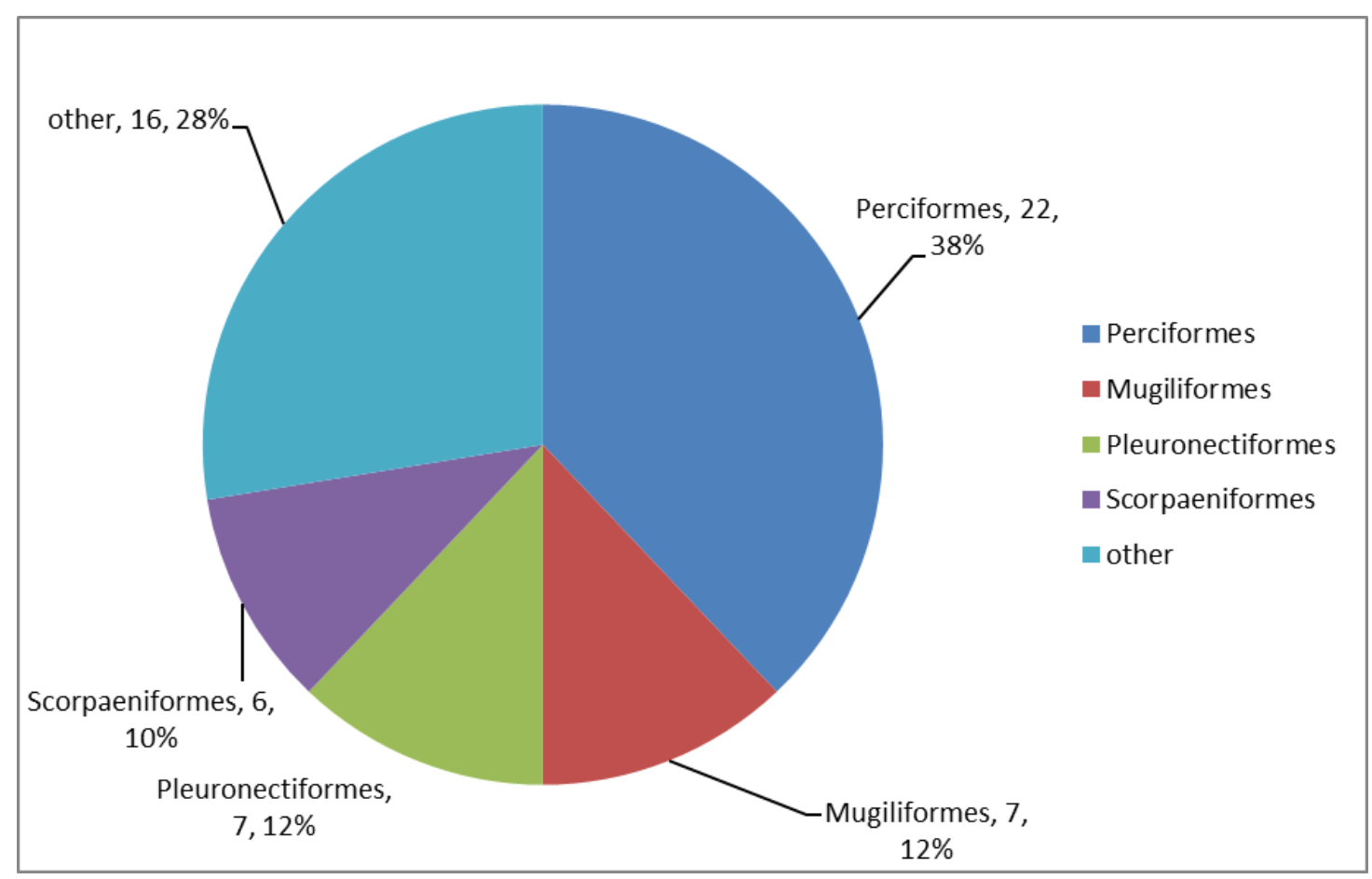

Figure 4: The host reported with Nerocila orbignyi according to their order.

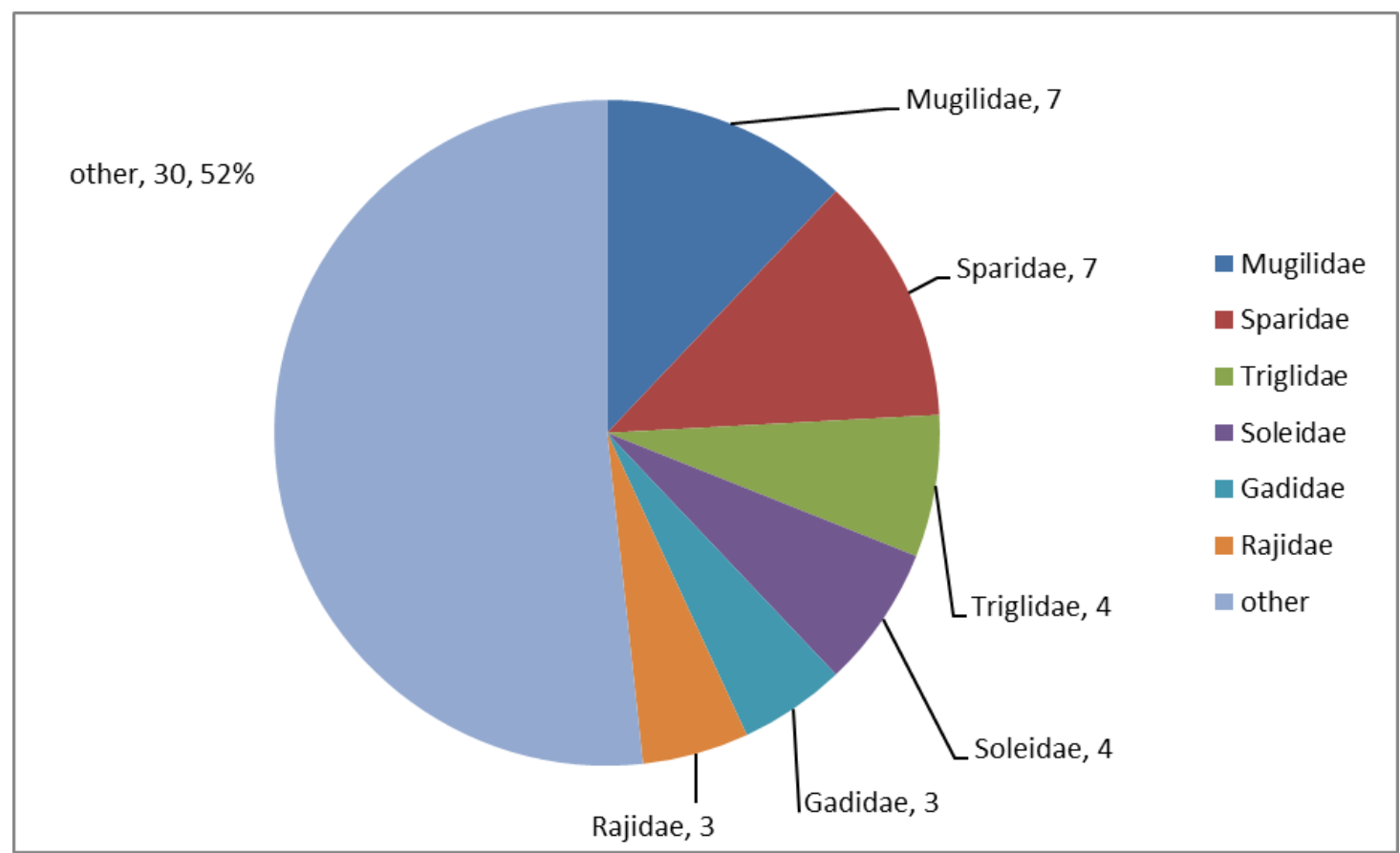

Figure 5: The host reported with Nerocila orbignyi according to their family. 


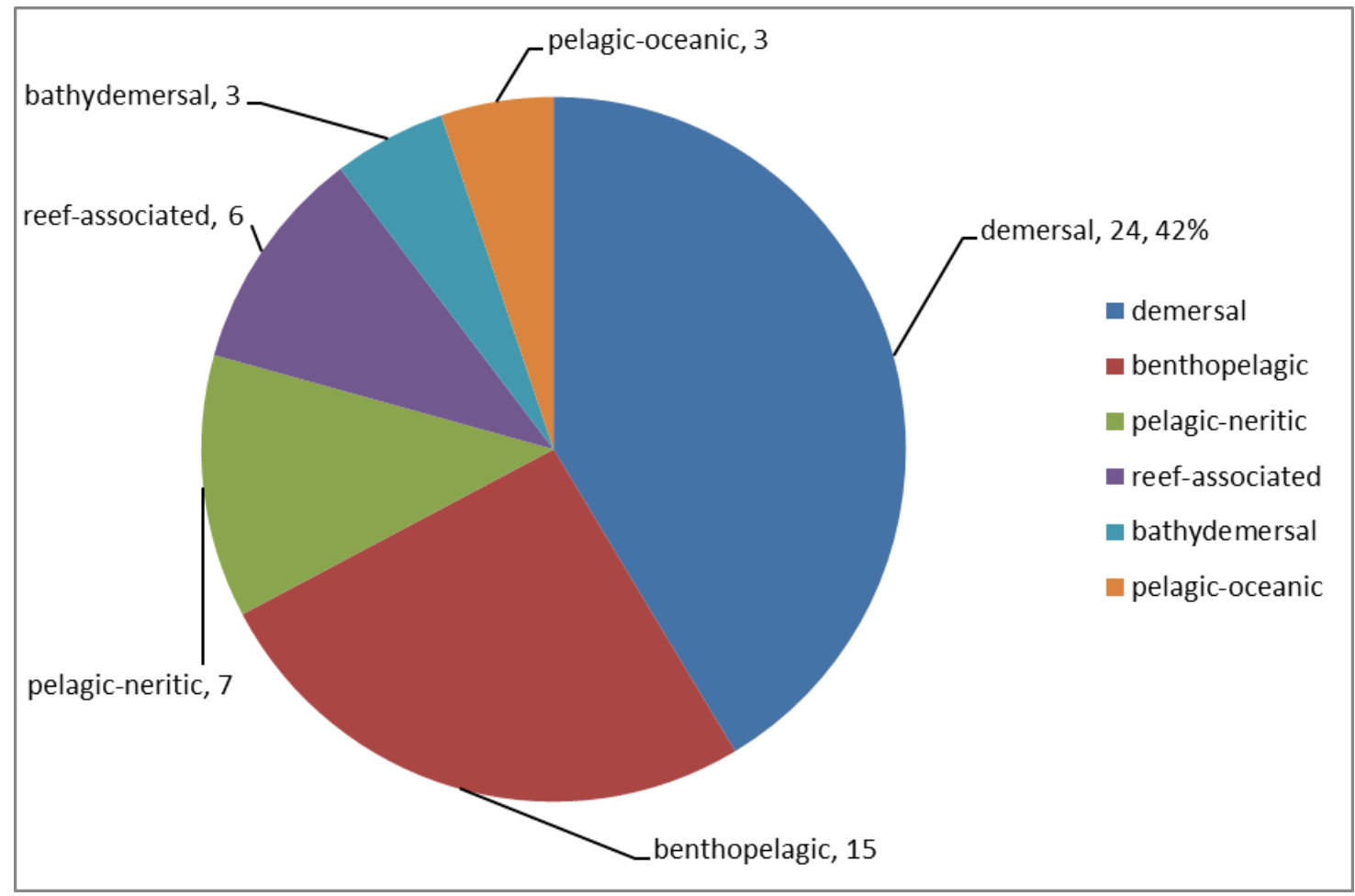

Figure 6: The host reported with Nerocila orbignyi according to habitat selections.

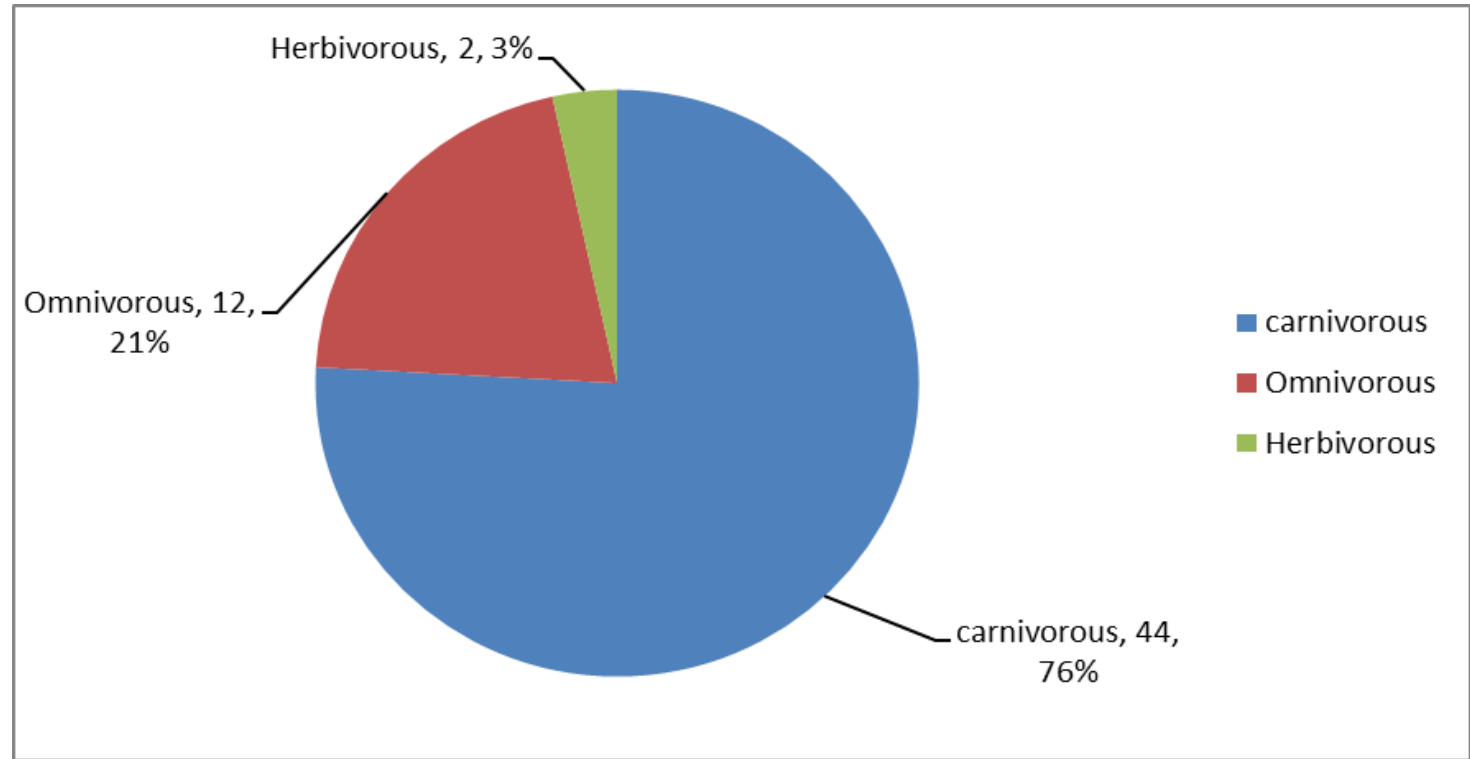

Figure 7: The host reported with Nerocila orbignyi according to feeding habits.

According to their solitary/schools; 33 (57\%) of 58 host fish species are schooling, 25 species (43\%) are solitary (Fig. 8). Although there is not much difference, it may be argued that Nerocila orbignyi is reported more frequently than fish that form a school. Luque et al. (2014) indicated that fish parasites prefer fish that form more schools than solitary fish especially ectoparasites. Brusca (1981) determined preferred hosts of Nerocila acuminata are schooling fishes. 


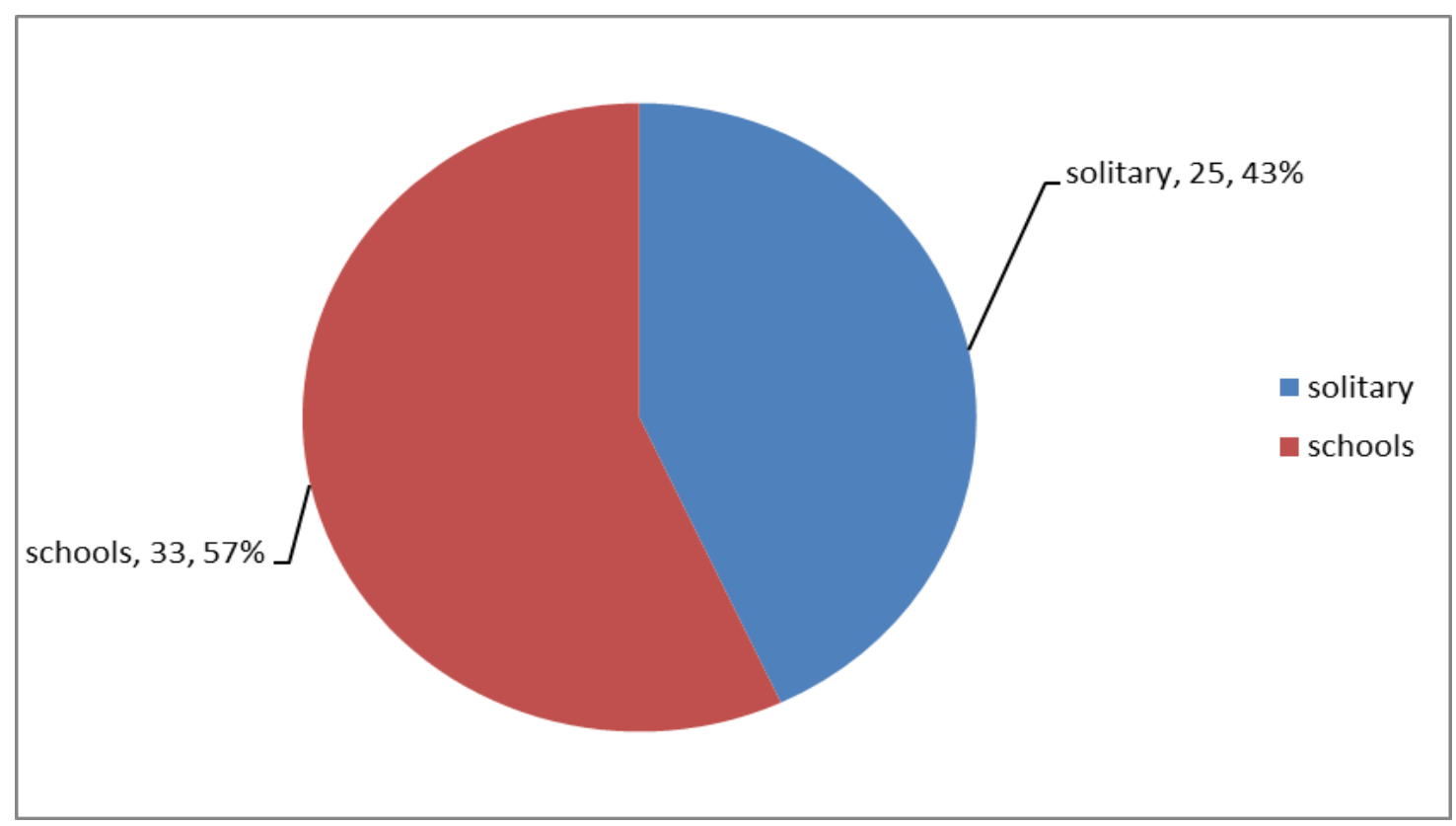

Figure 8: The host reported with Nerocila orbignyi according to solitary/schools.

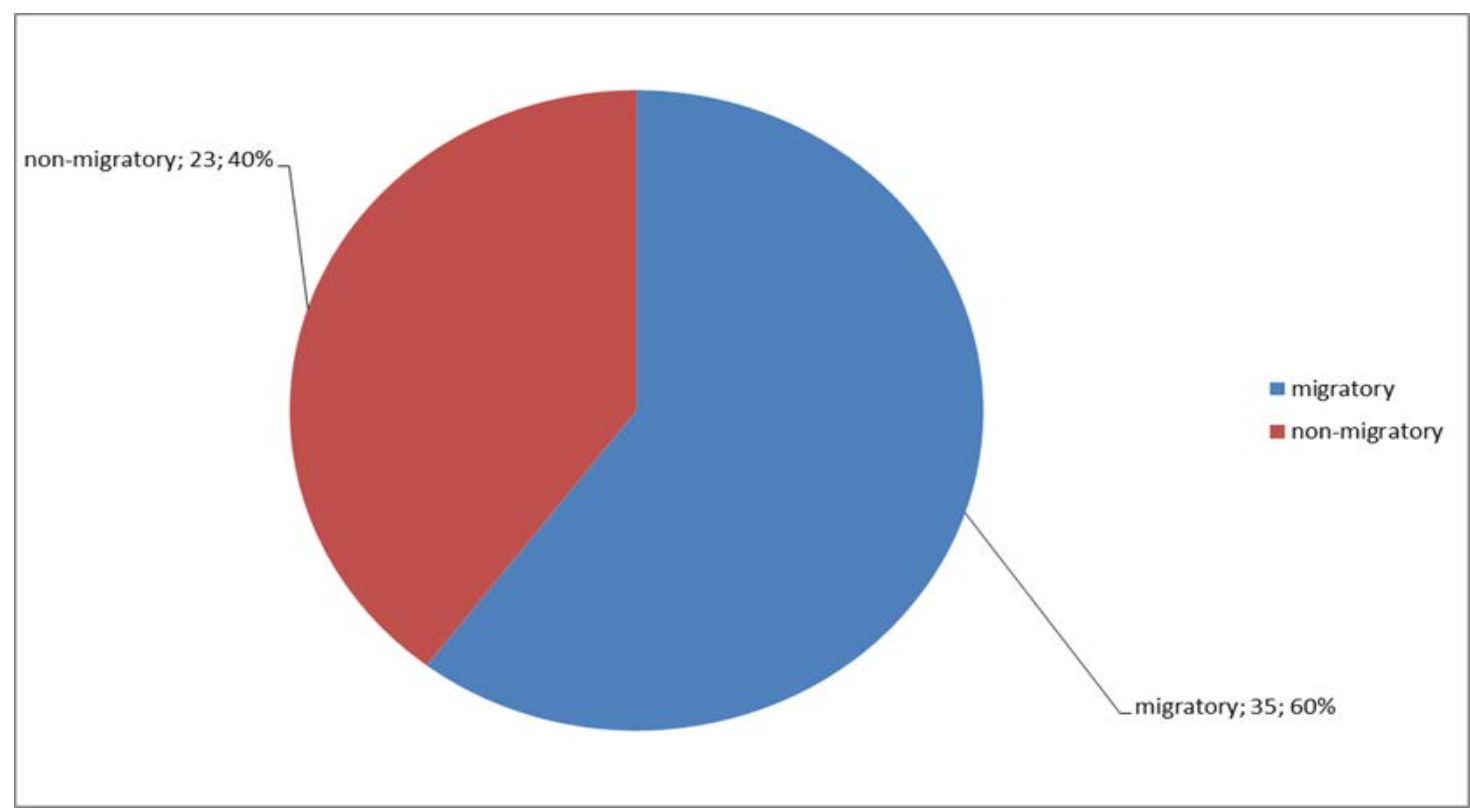

Figure 9: The host reported with Nerocila orbignyi according to migratory/non-migratory.

According to migratory/non-migratory; 35 species (60\%) of 58 host fish species are migratory; 23 species (40\%) to non-migratory. (Fig. 9). Fryer (1966) and Welicky and Sikkel (2015) founded that diurnal movements and nocturnal migrations of fish has an important role for the most settlement of parasite.

According to their scale types; 27 (46\%) of 58 host fish species are cycloid; 26 species (45\%) ctenoid; five species (9\%) with placoid (Fig. 10). 


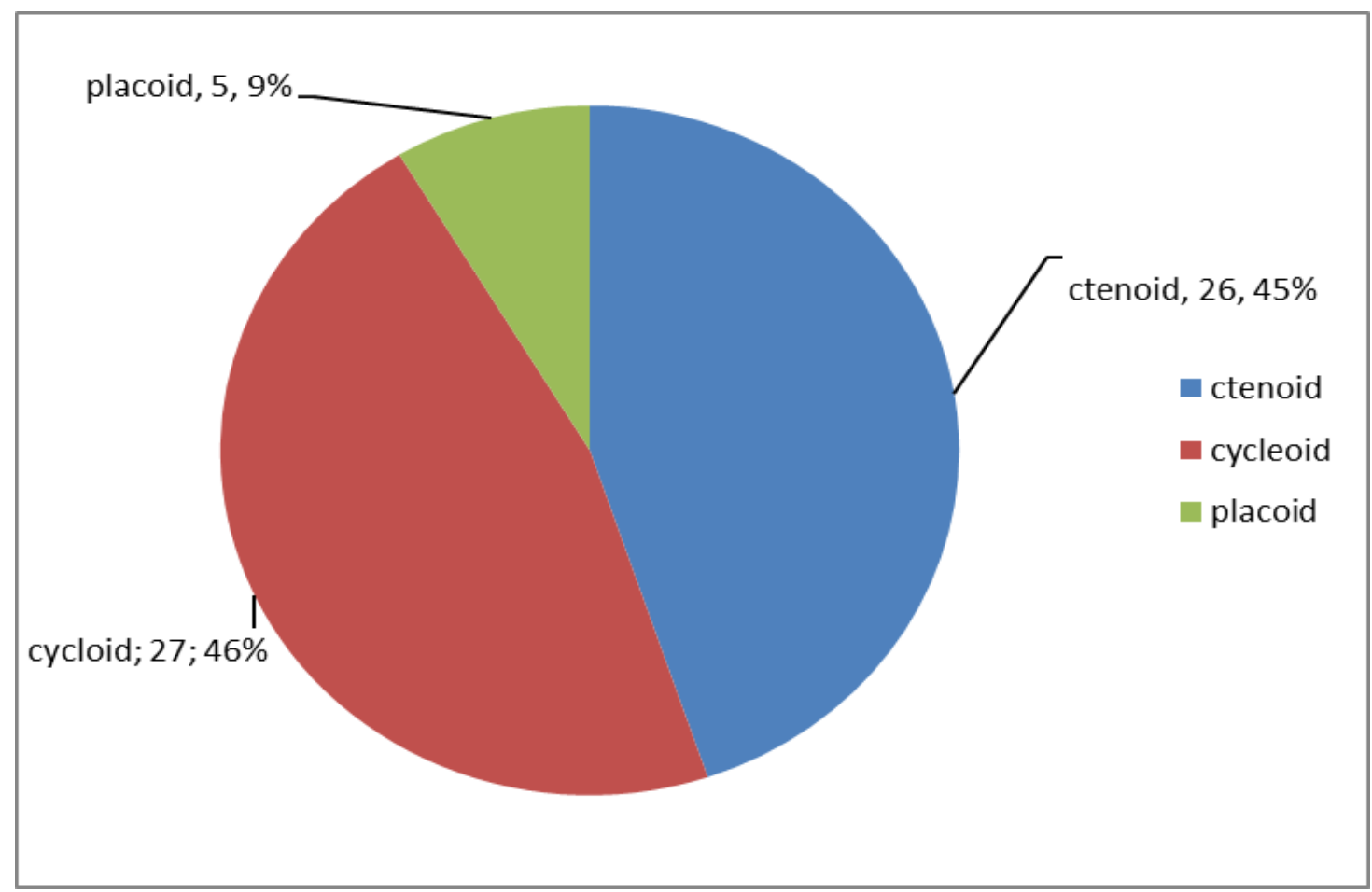

Figure 10: The host reported with Nerocila orbignyi according to scale type.

It may be said that Nerocila orbignyi has been reported more frequently than fish belonging to the Perciformes order and Mugilidae and Sparidae families. It may also be said that this parasite selects also the fishes with carnivorous, demersal, schools, and migratory character.

\section{CONCLUSIONS}

Cymothoids are an important group of parasites reported in fish. Nerocila is also a highly reported genus among Cymothoidae. It is a genus that is reported only from the body surface and fins in fish. Articles about cymothoid parasites are mostly in the form of reports and not much information is given about their morphology. The aim of this study is to give the updated morphology of Nerocila orbignyi and to provide comparison opportunities for those working on this subject. In addition, there is not much work on the host and host properties of cymothoid parasites. In this article, it is aimed to examine the host selectivity by mentioning both the morphology and ecology of the hosts of Nerocila orbignyi. 


\section{ACKNOWLEDGEMENTS}

The author would like to thank the editors, Dr. Angela Curtean-Bănăduc and Dr. Doru Bănăduc of this publication, for their continuous support. First author gratefully acknowledge Dr. Mike Robertson for technical and scientific support. 


\section{REFERENCES}

1. Adema J. P. H. M. and Huwae P. H. M., 1982 - New and supplementary records of marine Isopoda for the Netherlands and the Southern North Sea since 1956, with a note on Peltogaster paguri (Crustacea, Cirripedia), Zoologische Bijdragen, 28, 33-57.

2. Al-Zubaidy A. B. and Mhaisen F. T. 2013 - The first record of three Cymothoid Isopods from Red Sea fishes, Yemeni coastal waters, International Journal of Marine Science, 3, 166-172.

3. Balcells E., 1953 - Sur des isopodes, parasites de poissons, Vie et Milieu, 4, 547-552. (in French)

4. Berner L., 1969 - Les principaux Cymothoides (Crustaces, Isopodes) du Golfe de Marseille, Bulletin du Museum d'istoire Naturelle de Marseille, 29, 93-95. (in French)

5. Bragoni G., Romestand B. and Trilles J. P., 1983 - Cymothoadian parasitosis of the sea-dace (Dicentrarchus labrax Linnaeus 1758) during breeding, II, Parasitic ecophysiology in the Diana pond (upper Corsica), Annales de Parasitologie Humaine et Comparee, 58, 593-609.

6 Bilecenoğlu M., Kaya M., Cihangir B. and Çiçek E., 2014 - An updated checklist of the marine fishes of Turkey, Turkish Journal of Zoology, 38, 901-929.

7. Bragoni G., Romestand B. and Trilles J.P., 1984 - Parasitoses a Cymothoadien chez le loup, Dicentrarchus Labrax (Linnaeus, 1758), in Élevage, I, Écologie parasitaire dans le cas de L'etang de Diana (Haute-Corse) (Isopoda, Cymothoidae), Crustaceana, 47, 44-51 (in French)

8. Bruce N. L., 1987 - Australian species of Nerocila Leach, 1818, and Creniola n. gen. (Isopoda: Cymothoidae), crustacean parasites of marine fishes, Records of the Australian Museum, 39, 355-412.

9. Brusca R.C., 1978 - Studies on the Cymothoid fish symbionts of the eastern Pacific (Isopoda, Cymothoidae), I, Biology of Nerocila californica, Crustaceana, 34, 141-154

10. Castello J., Bitar G. and Zibrowius H., 2020 - Isopoda (crustacea) from the Levantine Sea with comments on the biogeography of Mediterranean isopods, Mediterranean Marine Science, 21/2, 308-339.

11. Cavaleiro F. and Santos M. J., 2007 - Survey of the metazoan ectoparasites of the European flounder Platichthys flesus (Linnaeus, 1758) along the north-central Portuguese coast, Journal of Parasitology, 93, 5, 1218-1222.

12. Charfi-Cheikhrouha F., Zghidi W., Oould Yarba L. and Trilles J. P., 2000 - Les Cymothoidae (isopodes parasites de poissons) des côtes tunisiennes: écologie et indices parasitologiques, Systematic Parasitology, 46, 146-150. (in French)

13. Châari M., Feki M. and Neifar L., 2015 - Metazoan parasites of the Mediterranean Garfish Belone belone gracilis (Teleostei: Belonidae) as a tool for stock discrimination, Open Journal of Marine Science, 5, 324-334.

14. Člak S., Kolega M., Mejdandžić D., Župan I., Šarić T., Piplović E. and Mustać B., 2018 Prevalence and effects of the cymothoid isopod (Ceratothoa oestroides, Risso 1816) on cultured meagre (Argyrosomus regius, Asso 1801) in the Eastern Adriatic Sea, Aquaculture Research, 49, 2, 1001-1007

15. Dollfus R. P. and Trilles J. P., 1976 - A propos de la collection Dollfus R. P., mise au point sur les Cymothoadiens jusqu’ à présent récoltés sur des Téléostéens du Maroc et de l'Algérie, Bulletin du Museum national d' Histoire naturelle de Paris, 272, 821-830. (in French)

16. Dorman J. A. and Holmes J. M. C., 1991 - Crustacean Ectoparasites of the Garfish Belone belone (L.) from Courtmacsherry Bay, Co Cork, The Irish Naturalists' Journal, 23, 10, 419423.

17. Er A. and Kayiş Ş., 2015 - Intensity and prevalence of some crustacean fish parasites in Turkey and their molecular identification, Turkish Journal of Zoology, 39, 1142-115. 
18. Fain-Maurel M.A., 1966 - Contribution a l'histologie et a la caryologie de quelques Isopodes, Spermiogenese et infrastructure du spermatozoide des Oniscides et des Cymothoides, A. J. et pl. I-X, Publie dans: Annals Science Nature, 12. 8, These, Paris, 1-188. (in French)

19. Ferri J., Petric M., Matic-Skoko S. and Dulcic J., 2008 - New host record, black scorpionfish Scorpaena porcus (Pisces, Scorpaenidae) for Nerocila orbigny and Ceratothoa parallela (Crustacea, Isopoda, Cymothoidae), Acta Adriatica, 49, 3, 255-258.

20. Fryer G., 1966 - Habitat selection and gregarious behaviour in parasitic crustaceans, in Crustaceana, 10, 199-209.

21. González L., Taylorb R. S., Bridlec A. R., Crosbiec P. B. B. and Nowak B. F., 2019 - Parasitic isopods Ceratothoa banksii (Leach, 1818) and Nerocila orbignyi (Guérin-Méneville, 1832) of farmed Atlantic salmon and their potential as vectors of Neoparamoeba perurans (Young et al. 2007) in Tasmania, Aquaculture, 507, 28-34.

22. Güven A. and Öztürk T., 2019 - Metazoan parasites of common sole (Solea solea) and scald fish (Arnoglossus laterna) (Pleuronectiformes) from Sinop Coast of Black Sea, Helminthologia, 56, 132-140.

23. Hadfield K. A., Coetzee A. and Smit N. J., 2013 - Ectoparasitic Nerocila Leach 1818 (Isopoda, Cymothoidae, Crustacea) species of South African fishes, the 42nd Annual Congress of the Parasitological Society of Southern Africa (PARSA) 22-24 September 2013, Stonehenge in Africa, Parys, Free State, South Africa.

24. Hale H. M., 1926 - Review of Australian isopods of the Cymothoid group, 11, Transactions and proceedings of the Royal Society of South Australia, 50, 201-234.

25. Hale H. M., 1940 - Report on the Cymothoid Isopoda obtained by the F. I. S. Endeavour on the coasts of Queensland, New South Wales, Victoria, Tasmania, and South Australia. Transactions and proceedings of the Royal Society of South Australia, 64, 2, 288-304.

26. Haswell W. A., 1880 - On some new Australian marine Isopoda, I, Proceedings of the Linnean Society of New South Wales, V, 470-481.

27. Hine P. M., Jones J. B. and Diggles B. K., 2000 - A checklist of parasites of New Zealand fishes, including previously unpublished records, NIWA Technical Report, 75.

28. Holthuis L. B., 1978 - Cymothoide isopode vande Nederlandse Kust en de zuidelijke moordzee, Bijdragen tot de faunistiek van Nederland. V, Zoologische Bijdragen, Leiden, 23(3), 28-33. (in Dutch)

29. Horton T. and Okamura B., 2001 - Cymothoid isopod parasites in aquaculture: a review and case study of a Turkish sea bass (Dicentrarchus labrax) and sea bream (Sparus auratus) farm, Diseases of Aquatic Organisms, 47, 181-188.

30. Horton T. and Okamura B., 2003 - Post-haemorrhagic anaemia in sea bass, Dicentrarchus labrax L., caused by blood feeding of Ceratothoa oestroides (Isopoda: Cymothoidae), Journal of Fish Diseases, 26, 401-406.

31. Horton T. and Baillie C., 2019 - Cymothoid isopods in UK Waters, Porcupine Marine Natural History Society Bulletin, 11, 44-50

32. Kayış Ş. and Ceylan Y., 2011 - First report of Nerocila orbigyni (Crustacea, Isopoda, Cymothoidae) on Solea solea (Teleostei, Soleidae) from Turkish Sea, Turkish Journal of Fisheries and Aquatic Sciences, 11, 167-169.

33. Kayış Ş., Er A., Kaçar Z. Z. and Ceylan Y., 2017 - The Cymothoid Isopod “Nerocila sp.” on fishes in Turkey, The 3rd International Symposium on EuroAsian Biodiversity 05-08 July 2017, Minsk, Belarus.

34. Kensley B. 1978 - Guide to the marine isopods of southern Africa, The Rustica Press (Pty.) Ltd. Trustees of the South African Museum, Wynberg, Cape Town, 173. 
35. Lemos de Castro A. and Gomes Corrêa M. M., 1982 - Sobre un curioso caso de ectoparasitismo de Telotha henselii (von Martens) (Isopoda-Cymotoidae) em Palaemonetes argentinus Nobili (Decapoda-Palaemonidae), Boletim do Museu Nacional Brasil, Zoology, 303, $1-8$.

36. Luque J. L., Mouillot D. and Poulin R., 2004 - Parasite biodiversity and its determinants in coastal marine teleost fishes of Brazil, Parasitology, 128, 671-682

37. Mahmoud N. E., Abdelwahab A. M., Khatab M. A. M. M. S. and Ramadan R. M., $2017-$ Further studies on Cymothoid Isopods of some fish species from lake Qarun Egypt, Egyptian Veterinary Medical Society of Parasitology Journal, 13, 15-24.

38. Marques J. F., Santos M. J., Costa J. L., Costa M. J. and Cabral H. N., 2005 - Metazoan parasites as biological indicators of population structure of Halobatrachus didactylus on the Portuguese coast, Journal of Applied Ichthyology, 21, 220-224.

39. Marques J. F., Santos M. J. and Cabral H. N., 2009 - Zoogeographical patterns of flatfish (Pleuronectiformes) parasites in the north-east Atlantic and the importance of the Portuguese coast as a transitional area, Scientia Marina, 73, 461-471.

40. Merella P. and Garippa G., 2001 - Metazoan parasites of grey mullets (Teleostea: Mugilidae) from the Mistras Lagoon (Sardina, western Mediterranean), Scientia Marina, 65, 201-206.

41. Mladineo I., 2002 - Prevalence of Ceratothoa oestroides (Risso, 1826), a cymothoid isopod parasite, in cultured sea bass Dicentrarchus labrax L. on two farms in the middle Adriatic Sea, Acta Adriatica, 43, 97-102.

42. Monod T., 1933 - Tanaidacea et Isopoda, in Mission Robert Ph. Dollfus en Egypte, Memories of Institute of Egypte, XXI, 161-264. (in French)

43. Montero F. E., 2001 - Estudio parasitológico en cultivos de Seriola dumerili (Risso, 1810) en el Mediterráneo, Tesis doctoral. Universidad de Valencia. (in Spanish)

44. Moreira P. S. and Sadowsky V., 1978 - An annotated bibliography of parasitic Isopoda (Crustacea) of Chondrichthyes, Boletim do Instituto Oceanográfico, S Paulo, 27, 95-152.

45. Northcott S. J., Lyndon A. R. and Campbell A. D., 1997 - An outbreak of freshwater fish lice, Argulus foliaceus L., seriously affecting a Scottish stillwater fishery, Fisheries Management and Ecology, 4, 73-75.

46. Öktener A. and Trilles J. P., 2004 - Report on the cymothoids (Crustacea, Isopoda) collected from marine fishes in Turkey, Acta Adriatica, 45, 145-154.

47. Öktener A., Tuncer S. and Trilles J. P., 2020 - Nerocila milesensis n. sp. (Isopoda: Cymothoidae) parasitic on Devil Firefish Pterois miles (Bennett, 1828) from the South Aegean Sea, Turkey, Vie et milieu - Life and environment, 70, 1, 7-17

48. Öktener A. and Ünal A., 2020 - Infestation of Fish louse, Argulus foliaceus (Linnaeus, 1758) (Crustacea: Branchiura) on Rainbow Trout farm in Manyas Dam Lake, Turkey, Journal of Wetlands Biodiversity, 10, 95-111

49. Özcan T., Kirkim F. and Sakalı U., 2015 - Serranus cabrilla (Linnaeus, 1758) (Perciformes, Serranidae) a new host record for Nerocila orbignyi (Guérin-Mèneville, 1832) (Isopoda, Cymothoidae), Iranian Journal Fisheries Sciences, 14, 4, 1083-1088.

50. Panarese R., Tedesco P., Chimienti G., Latrofa M. S., Quaglio F., Passantino G., Buınavoglia C., Gustinelli A., Tursi A. and Otranto D. 2019 - Haplosporidium pinnae associated with mass mortality in endangered Pinna nobilis (Linnaeus 1758) fan mussels, Journal of Invertebrate Pathology, 164, 32-37.

51. Paperna I. and Overstreet R., 1981 - Parasites and Diseases of Mullets (Mugilidae), Faculty Publications from the Harold W. Manter Laboratory of Parasitology, 579, 411-493.

52. Potter I. C., Gardner D. C. and Claridge P. N., 1988 - Age composition, growth, movements, meristics and parasites of the whiting, Merlangius merlangus, in the Severn Estuary and Bristol Channel, Journal of the Marine Biological Association of the United Kingdom, 68, 295-313. 
53. Ramdane Z., Bensouilah M. A. and Trilles J. P., 2007 - The Cymothoidae (Crustacea, Isopoda), parasites on marine fishes, from Algerian fauna, Belgian Journal of Zoology, 137, 1, 67-74.

54. Rokicki J., 1985 - Biology of adult Isopoda (Crustacea) parasitizing fishes of North-West Africa shelf, Acta Ichthyologica et Piscatoria, 15, 1, 95-122.

55. Rokicki J., 1985 - Biology of adult Isopoda (Crustacea) parasitizing fishes of North-West Africa shelf, Acta Ichthyologica et Piscatoria, 15, 1, 95-122.

56. Rokicki J., 1985 - Biology of adult Isopoda (Crustacea) parasitizing fishes of North-West Africa shelf, Acta Ichthyologica et Piscatoria, 15, 1, 95-122.

57. Scott D., 1964 - Parasitic isopods on trout, Proceedings of the New Zealand Ecological Society $11,23$.

58. Shaheen A. A., Abd el Latif A. M., Elmadawy R. S. and Noor Eldeen A. I., 2017 - Isopodiosis in some fishes from Egyptian Qaroun Lake: prevalence, identification, pathology and in vitro trials to get rid of it, Research Journal of Pharmaceutical, Biological and Chemical Sciences, 8, 1, 1971-1979.

59. Stadler T., 1972 - Braga bachmanni, nuevo ectoparasito de Ancistrus cirrosus (Crustacea, Cymothoidae) en pisces Loricariidae, Neotropica, 18, 141-145. (in Spanish)

60. Thatcher V. E., 1995 - Comparative pleopod morphology of eleven species of parasitic isopods from Brazilian fish, Amazoniana, XIII, 3/4, 305-314.

61. Trilles J. P., 1961 - Sur Nerocila orbignyi schioedte et Meinert, 1881 (Isopoda, Cymothoidae) du bassin Occidental de la Mediterranee. 86 eme Congress des Societes Savantes, 689-696. (in French)

62. Trilles J. P., 1962 - Remarques morphologiques sur les "Isopodes Cymothoidae” parasites de Poissons de l’etang de Thau, Naturalia Monspeliensis, III, Zoologie, 101-124. (in French)

63. Trilles J. P., 1964a - Specificite parasitaire chez les Isopodes Cymothoidae, mediteraneens. Note preliminaire, Vie et Milieu, XV, 105-116. (in French)

64. Trilles J. P., 1964b - Variations morphologiques du Crane chez les Teleosteens Sparidae et Centracanthidae en rapport avec l'existence chez ces poissons de certains Cymothoidae parasites, Annales de Parasitologie, XXXIX, 5, 627-630. (in French)

65. Trilles J. P., 1968 - Recherches sur les Isopodes Cymothoidae des côtes françaises. L Systématiques et faunistiques. I. Bionomie et parasitisme, 2: hèse de Doctoratès Sciences, Montpellier, enregistrement C. N. R. S. A. O., 2305, 1181, 139, photographies 156, 1397. (in French)

66. Trilles J. P., 1975 - Les Cymothoidae (Isopoda, Flabellifera) des côtes françaises, II, Les Anilocridae Schioedte et Meinert, 1881. Genres Anilocra Leach, 1818 et Nerocila Leach, 1818, Bulletin du Muséum national d'Histoire naturelle, Paris, 3, 347-378. (in French)

67. Trilles J. P. 1977 - Les Cymothoidae (Isopoda, Flabellifera; parasites de poissons) du Rijksmuseum van Natuurlijke Historie de Leiden, Méditerranée et Atlantique, Zoologische Mededelingen Leiden, 52, 7-17. (in French)

68. Trilles J. P., 1994 - Les Cymothoidae (Crustacea, Isopoda) du Monde (Prodrome pour une Faune), Studia Marina, 21/22, 1-288. (in French)

69. Trilles J. P. and Raibaut A., 1973 - Sur les Cymothoidae (Isopoda, Flabellifera) parasites de poissons marins de Tunisie, 2nd Note, Bulletin du Muséum national d'Histoire naturelle, 3, 144, 273-281. (in French)

70. Trilles J. P, Radujkovic B. M. and Romestand B., 1989 - Parasites de poissons marins du Montenegro: isopodes, in Radujkovic B. M. and Raibaut A. (eds). Faune des parasites des poissons marins des côtes du Montenegro (Adriatique sud), Acta Adriatica, 30, 279-306. (in French)

71. Webb B. F., 1973 - Fish populations of the Avon-heathcote estuary, New Zealand Journal of Marine and Freshwater Research, 7, 301-305. 
72. Welicky R. L. and Sikkel P. C., 2015 - Decreased movement related to parasite infection in a diel migratory coral reef fish, Behavioral Ecology and Sociobiology, 69, 1437-1446.

73. WoRMS Editorial Board, 2020 - World Register of Marine Species, http://www.marinespecies.org at VLIZ.

74. Wunder S. W., 1962 - Nerocila orbignyi, ein protandrischer hermaphrodit und parasitischer isopode auf Tilapia galilaea im see Borullus in Egypten, Verhandlungen der Deutschen Zoologischen Gesellschaft, 22, 140-151. (in German) 